Original Research Paper

\title{
Computer Aid Screening for Potential Antimalarial Choroquinone Compounds as Covid 19 Utilizing Computational Calculations and Molecular Docking Study
}

\author{
${ }^{1}$ Asmaa Aboelnaga, ${ }^{2}$ Asmaa M. Fahim and ${ }^{1,3}$ Taghreed H. EL-Sayed \\ ${ }^{1}$ Department of Chemistry, Faculty of Women of Arts, Science and Education, Ain Shams University, Heliopolis, Egypt \\ ${ }^{2}$ Departments of Green Chemistry, National Research Center, Dokki, P.O. Box.12622 Cairo, Egypt \\ ${ }^{3}$ Departments of Chemistry, Faculty of Science, Taibah University, Yanbu, Saudi Arabia
}

\author{
Article history \\ Received: 28-09-2020 \\ Revised: 25-11-2020 \\ Accepted: 30-11-2020 \\ Corresponding Author: \\ Asmaa Aboelnaga \\ Department of Chemistry, \\ Faculty of Women of Arts, \\ Science and Education, Ain \\ Shams University, Heliopolis, \\ Egypt \\ Email: \\ asmaa.aboelnaga@women.as \\ u.edu.eg \\ And \\ Asmaa M Fahim \\ Departments of Green \\ Chemistry, National Research \\ Center, Dokki, P.O. Box.12622 \\ Cairo, Egypt \\ Email: \\ asmaamahmoud8521@hotmail. \\ com
}

\begin{abstract}
Click reaction of 4,7-dichloroquinoline (1) with thiosemicarbazide (2) to afford the corresponding 2-(7-chloroquinolin4-yl)thiosemicarbazide (3) utilized ultrasonic irradiation which can cyclized easily with ethylacetoacetate to give the 3-(7-chloroquinolin-4ylamino)-tetrahydro-6-methyl-2-thioxopyrimidin-4(1H)-one (4) via nucleophilic substitution reaction. The synthesized compounds was examined in vitro antimalarial activity with $\mathrm{IC}_{50}=11.92,25.37 \mu \mathrm{g} / \mathrm{mL}$ against chloroquinone drug. Furthermore, the theoretical investigation of most active compounds CQT and CQP utilizing of DFT/B3LYP/6$311 \mathrm{G}(\mathrm{d})$ and HF/6-311G(d) basis's set and evaluated their physical characters, bond length, bond angles, dihedral angles, also its HOMOLUMO energy gap was $3.77 \mathrm{eV}$ which indicate the reactivity of CQP. Moreover, the molecular docking studies of these synthesized compounds showed small energy affinity against SARS-CoV-2 main protease (PDBID: 6lu7) and crystal structure of thermoplasma acidophilum (PDBID: 1q2w) and shorter bond length. All these parameters could be considered with different extent to significantly affect the binding affinity of these compounds to the active protein sites for further biological evaluation on Covid-19.
\end{abstract}

Keywords: Covid 19, Novel Chloroquinone Compounds, Antimalarial Activity, Computational Studies, Docking Interaction

\section{Introduction}

In December 2019, several cases of pneumonia of an unknown etiology appeared in Wuhan, Hubei Province, China. Later, a novel coronavirus was identified in a bronchoalveolar lavage fluid sample from the Wuhan Seafood Market through the use of metagenomic nextgeneration sequencing technology (Yang and Wang, 2020; Wu et al., 2020a). On February 11, 2020, the International Committee on Taxonomy of Viruses (ICTV) named the virus Severe Acute Respiratory Syndrome Coronavirus-2 (SARS-CoV-2). This is the seventh member of the coronavirus family that can infect humans after the appearance of Severe Acute Respiratory Syndrome Coronavirus (SARS-CoV) and
Middle East Respiratory Syndrome Coronavirus (MERS-CoV). The World Health Organization classified the coronavirus pneumonia epidemic caused by SARS$\mathrm{CoV}-2$ as a public health emergency of international concern on January 30, 2020 (Zhao et al., 2010; Zhou et al., 2020). A thorough program, including surveillance, diagnostics, clinical treatment, research and the development of vaccines and drugs, is crucial to win the battle against COVID-19 and other infectious diseases (Yang and Wang, 2020). Etiology of SARS-COV-2 Corona viruses are not new infectious pathogens in the world. The first described coronavirus was isolated from chickens in 1937 (Ludwig and Zarbock, 2020). In the mid-1960s the first human coronaviruses were first identified (Steardo et al., 2020). The Corona virus family 
can be divided into four genera: $\alpha, \beta, \gamma$ and $\delta$, as per the genome structure and phylogenetic analysis of coronaviruses (Fehr and Perlman, 2015). The coronaviruses of the $\alpha$ and $\beta$ mainly infect mammals and humans, while the coronaviruses of the $\gamma$ and $\delta$ typically infect birds (Rodriguez-Morales et al., 2020; Guo et al., 2020). The pathogen that is causing the pandemic is related to the Acute Respiratory Syndrome Coronavirus (SARS-CoV), which caused another back outbreak in 2003 (Sjödin et al., 2020; Mirza and Froeyen, 2020). As of yet, there are no effective treatments or targeted therapeutics against the virus. Because of that, the scientific community is striving to investigate many different mechanisms to interfere with the virus' metabolism. Consequently, in recent clinical trials against COVID-19 several antiviral drugs used with patients with similar viral infections to antimalarial drugs, such chloroquine (I), quinine (II) and amodiaquine (III) in malaria treatment is common (dos Santos Chagas et al., 2019; Kwofie et al., 2020). Also the hydroxychloroquine (IV) has approved drug for malaria disease treatment via the FDA was explored as a medication for SARS-CoV-2. As displayed in Figure 1 (Yao et al., 2020; Liu et al., 2020). Previous reports have shown that, the chloroquine and hydroxychloroquine can inhibit the Coronavirus (COVID-19) by altering the $\mathrm{pH}$ at the surface of the cell membrane. This can inhibit the attachment of the virus to the cell membrane. Furthermore, it can prevent nucleic acid replication, glycosylation of viral proteins, virus assembly, new virus particle delivery, virus release and other mechanisms to obtain its antiviral effects (Grimstein et al., 2019).
The theoretical DFT calculations and the conformational analyses can result in a great contribution to drug discovery by reducing financial costs and saving time (especially for emerging diseases such as COVID-19 and speeding up analyses of target interactions with drug candidates (Gimeno et al., 2019). As a result, different computational studies have been published to help better understand the mechanism of M-pro and try to inhibit its function (Mirza and Froeyen, 2020; Ton et al., 2020; Kong et al., 2020; Tang et al., 2020; Chen et al., 2020; Liu and Wang, 2020; Adem et al., 2020; Yoshino et al., 2020; Hosseini and Amanlou, 2020; Bzówka et al., 2020a; 2020b; Khaerunnisa et al., 2020; Xu et al., 2020).

In this investigation, we concern in our work of theoretical determination of the molecular geometry of synthesized materials 2-(7-chloroquinolin-4yl)thiosemicarbazide (3) (CQT) and 3-(7-chloroquinolin4-ylamino)-tetrahydro-6-methyl-2-thioxopyrimidin4(1H)-one (Aboelnaga and EL-Sayed, 2018) (4) (CQP) which exhibited excellent antimalarial activity against chloroquinone drug. Our stimulation was focused on confirmation of our compounds and comparison with 4,7-dichloroquinoline from physical properties, bond length, bond angles utilizing DFT/B3LYP/6-311G(d) and HF/6-311G(d) basis set (Hagar et al., 2020; Arif et al., 2020; Wu et al., 2020b). Furthermore, the molecular docking studies of these compounds against target main protease (Mpro) of SARS-CoV-2: Mpro (PDBID: 6lu7) and crystal structure of thermoplasma acidophilum (PDBID: 1qw2) to know the binding interaction energy and interaction hydrogen bonding between our compounds and the different protein's sites.

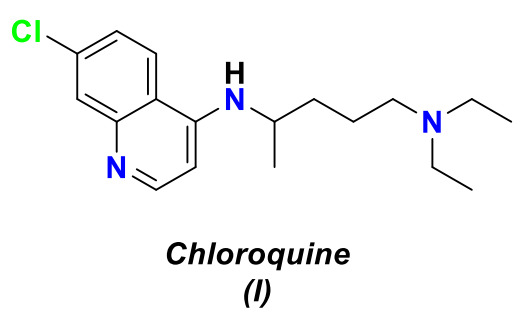<smiles>C=C[C@H]1CN2CC[C@@H]1C[C@H]2[C@H](O)c1ccnc2ccc(OC)cc12</smiles><smiles>CCN(CC)Cc1cc(Nc2ccnc3cc(Cl)ccc23)ccc1O</smiles><smiles>CCN(CCO)CCCC(C)Nc1ccnc2cc(Cl)ccc12</smiles>

Hydroxychloroquine (IV)

Fig. 1: Some of antimalarial dugs attached to quinolone ring 


\section{Results and Discussion}

\section{Chemistry}

The nucleophilic substitution reaction 4,7dichloroquinoline (1) with thiosemicarbazide (2) utilizing ultrasonic irradiation for $30 \mathrm{~min}$ at room temperature to afford the corresponding 2-(7chloroquinolin-4-yl) thiosemicarbazide (3) as Click reaction as displayed in Fig. 2. The obtained compound was investigated via spectral data as; the spectrum of IR of the was displayed vibrational bands at amino and $\mathrm{NH}$ group at $v=3390 \mathrm{~cm}^{-1}, v=3150 \mathrm{~cm}^{-1}$; respectively, while the $\mathrm{NH}$ bending vibration was showed at $v=1585 \mathrm{~cm}^{-1}$. Furthermore, the ${ }^{1} \mathrm{HNMR}$ of the investigated novel thiosemicarbazide was showed signals at $\delta 8.89$ due to $\mathrm{N}=\mathrm{CH}$, quinolone and singlet signal of $\mathrm{NH}_{2}$ at $\delta 8.20$. The mass spectrum showed a peak at $\mathrm{m} / \mathrm{z} 252$ corresponding to its molecular ion (Aboelnaga and EL-Sayed, 2018).

The reactivity of 2-(7-chloroquinolin-4yl)thiosemicarbazide (3) with ethylacetoacetate utilizing ultrasonic irradiation for $40 \mathrm{~min}$ at $90^{\circ} \mathrm{C}$ to afford the corresponding 3-(7-chloroquinolin-4-ylamino)tetrahydro-6-methyl-2-thioxopyrimidin-4(1H)-one (4). The spectral data confirmed the obtained compound where its FT-IR spectroscopy showed stretching NH at $v$
$3110 \mathrm{~cm}^{-1}$ and bending vibration at $1587 \mathrm{~cm}^{-1}$, also the $\mathrm{C}=\mathrm{O}$ band showed at $1674 \mathrm{~cm}^{-1}$ while the $\mathrm{C}=\mathrm{S}$ appeared at $1211 \mathrm{~cm}^{-1}$. The ${ }^{1} \mathrm{HNMR}$ was assigned the doublet signals of $\mathrm{N}=\mathrm{CH}$ quinolone at $\delta 8.20 \mathrm{ppm}$ and aromatic regions ranges between $\delta 7.15-7.85 \mathrm{ppm}$. The mass spectrum of the revealed compound showed $\mathrm{m} / \mathrm{z}=$ 318 (Aboelnaga and EL-Sayed, 2018).

\section{Computational Studies}

\section{Molecular Orbital Calculations}

The $\pi$-effects properties have an important contribution to biological systems that binding to the active site of a protein receptor so the most active optimized molecular structures of 2-(7-Chloroquinolin4-yl)thiosemicarbazide (3) (CQT) and 3-(7chloroquinolin-4-ylamino)-tetrahydro-6-methyl-2thioxopyrimidin-4(1H)-one (4) (CQP) is demonstrated in Fig. 3 and selected bond lengths, bond angles and dihedral angles are scheduled in Table 3 calculated through B3LYP/6-311G (d) The molecular structure of these compound were not planar (Akl et al., 2020; Fahim and Ismael, 2019; Trott and Olson, 2010). The optimized structures of CQT (3), $\mathrm{x}$ ray of 4,7dichloroquinoline (Hema et al., 2015) and CQP (4) as showed in Table 1 and Fig. 3.<smiles>Clc1ccc2c(Cl)cc[nH+]c2c1</smiles>

(1)<smiles>CCO[Mg]O[Na]</smiles>

(2)<smiles>C=C(N)N(N)c1ccnc2cc(Cl)ccc12</smiles>

(3)<smiles>CCOC(=O)CC(C)=O</smiles><smiles>CC1CC(=O)N(Nc2ccnc3cc(Cl)ccc23)C(=S)N1</smiles>

(4)

Fig. 2: (a) Ultrasonic reaction of 4,7-dichloroquinoline (1) with thiosemicarbazide (2); (b) Reactivity of 2-(7-chloroquinolin-4-yl) thiosemicarbazide (3) with ethylacetoacetate 


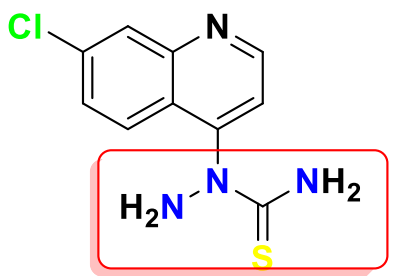

(CQT)

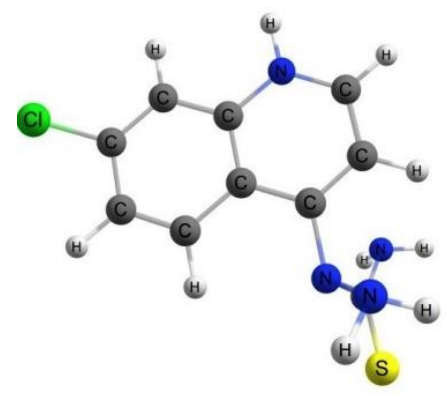

$C Q T$<smiles>Clc1ccc2c(Cl)ccnc2c1</smiles>

4,7-dichloroquinoline

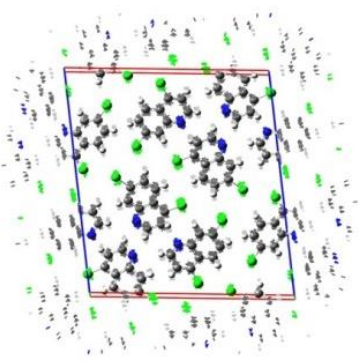

4,7-dichloroquinoline<smiles>CC1CC(=O)N(Nc2ccnc3cc(Cl)ccc23)C(=S)N1</smiles>

(CQP)

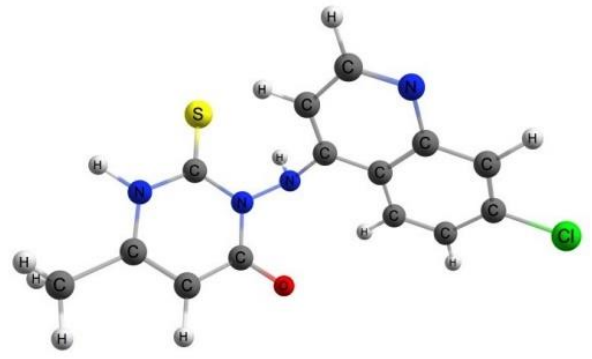

$C Q P$

Fig. 3: The optimized geometry, numbering system and moiety of Compounds CQT (3), X ray of 4,7-dichloroquinoline (Fahim and Shalaby, 2019) and CQP(4), utilizing of DFT/B3LYP/6-311(G)-d

The optimized structure of compound CQT (3) have a different bond lengths, bond angles and dihedral angles utilizing B3LYP/6-311G(d), HF/6-311G(d). Comparing the theoretical data with the crystallographic data 4,7dichloroquinoline (Hema et al., 2015) indicated slightly alteration in the optimized bond lengths and bond angles. It was noticed that $\mathrm{N}_{16}-\mathrm{C}_{11}$ of CQT (3) measured $1.37286 \AA, \quad 1.30236 \AA$ of $\mathrm{HF} / 6-311 \mathrm{G}(\mathrm{d}), \quad \mathrm{B} 3 \mathrm{LYP} / 6-$ $311 \mathrm{G}(\mathrm{d})$; respectively while the $\mathrm{x}$-ray of $\mathrm{N}_{1} \mathrm{~A}-\mathrm{C}_{9} \mathrm{~A}$

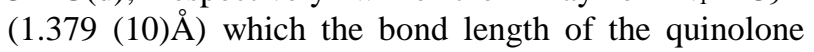
was compatible with HF theory, but the other bonds such as $\mathrm{C}_{5}-\mathrm{C}_{6}(1.7600 \AA),(1.4023 \AA)$ for $\mathrm{HF} / 6-311 \mathrm{G}(\mathrm{d})$, $\mathrm{B} 3 \mathrm{LYP} / 6-311 \mathrm{G}(\mathrm{d})$; respectively where it is compatible with $\mathrm{C}_{2} \mathrm{~A}-\mathrm{C}_{3} \mathrm{~A}$ (1.432 (12) $\left.\mathrm{A}\right)$. Furthermore, the bond angles of CQT (3) such as $\mathrm{N}_{16}-\mathrm{C}_{11}-\mathrm{C}_{12}\left(120.8622^{\circ}\right)$, (120.5236 ${ }^{\circ}$ ) for $\mathrm{HF} / 6-311 \mathrm{G}(\mathrm{d}), \mathrm{B} 3 \mathrm{LYP} / 6-311 \mathrm{G}(\mathrm{d})$ while the $\mathrm{x}$ ray bond of $\mathrm{C}_{6} \mathrm{~A}-\mathrm{C}_{5} \mathrm{~A}-\mathrm{C}_{10} \mathrm{~A}\left(120.2(8)^{\circ}\right)$ which is more nearest to $\mathrm{B} 3 \mathrm{LYP} / 6-311 \mathrm{G}(\mathrm{d})$, also $\mathrm{Cl}_{17}-\mathrm{C}_{6}-\mathrm{C}_{5}$ $\left(120.64566^{\circ}\right), \quad\left(122.0236^{\circ}\right)$ and the $\mathrm{C}_{3} \mathrm{~A}-\mathrm{C}_{4} \mathrm{~A}-\mathrm{Cl}_{1} \mathrm{~A}$ $\left(119.5(7)^{\circ}\right)$. Moreover, the dihedral angles of attached thiosemicarbazide $\quad \mathrm{H}_{20}-\mathrm{N}_{19}-\mathrm{N}_{18}-\mathrm{C}_{22} \quad\left(-109.7568^{\circ}\right)$, ($\left.108.235^{\circ}\right)$ and $\mathrm{N}_{19}-\mathrm{N}_{18}-\mathrm{C}_{22}-\mathrm{S}_{23}\left(126.7877^{\circ}\right),\left(128.2365^{\circ}\right)$ for HF and DFT/B3LYP basis set which meant that this group out of plane and more reactive but the dihedral angle $\mathrm{N}_{16}-\mathrm{C}_{4}-\mathrm{C}_{3}-\mathrm{C}_{9} \quad\left(-0.00075^{\circ}\right), \quad\left(-0.00072^{\circ}\right)$ which compatible with the $\mathrm{x}$-ray $\mathrm{C}_{9} \mathrm{~A}-\mathrm{N}_{1} \mathrm{~A}-\mathrm{C}_{2} \mathrm{~A}-\mathrm{C}_{3} \mathrm{~A}\left(0.9(12)^{\circ}\right)$ as shown in Table 1.

Also the optimized of CQP (4) and illustrated the bond lengths, angles and dihedral angles as displayed in
Table 1 and Fig. 3. The bond lengths of tetrahydro-6methyl-2-thioxopyrimidin-4(1H)-one were utilizing B3LYP/6-311G (d), HF/6-311G (d) theory level. The bond length of $\mathrm{N}_{17}-\mathrm{N}_{25}(1.4700 \AA)(1.5912 \AA),\left(\mathrm{C}_{19}-\mathrm{S}_{23}\right)$

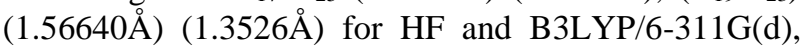
respectively which they were mostly same included that the more delocalization of charges on tetrahydro-6methyl-2-thioxopyrimidin-4(1H)-one with fixed bond length, while the smallest difference in bond length between theoretical and experimental of chloroquinone ring such as for $\mathrm{C}_{22}-\mathrm{C}_{21}$ for theoretical and for $\mathrm{C}_{5} \mathrm{~B}-\mathrm{C}_{6} \mathrm{~B}$ of experimental was $(0.0135 \AA)$ for DFT method. Also the largest difference between experimental and calculated DFT bond angles is $\mathrm{C}_{5}-\mathrm{C}_{6}-\mathrm{Cl}_{16}$ and $\mathrm{C}_{3} \mathrm{~B}-\mathrm{C}_{4} \mathrm{~B}-$ $\mathrm{Cl}_{1} \mathrm{~B}\left(1.3764^{\circ}\right)$. Therefore, the difference of bond angles of $\left(\mathrm{S}_{26}-\mathrm{C}_{19}-\mathrm{N} 27\right),\left(\mathrm{C}_{19}-\mathrm{N}_{27}-\mathrm{H}_{23}\right)$ between $\mathrm{HF}$ and DFT/B3LYP is $\left(1.22508^{\circ}\right),\left(3.24341^{\circ}\right)$. Additionally, the dihedral angles of tetrahydro-6-methyl-2thioxopyrimidin-4(1H)-one $\left(\mathrm{N}_{27}-\mathrm{C}_{22}-\mathrm{C}_{28}-\mathrm{H}_{29}\right),\left(\mathrm{N}_{17}-\mathrm{N}_{25}-\right.$ $\mathrm{C}_{19}-\mathrm{N}_{27}$ ) was taken a negative charges which were out of plane of chloroquinone and give stability of this moiety, also the dihedral of chloroquinone were compatible the theoretical and experimental $\left(\mathrm{C}_{6}-\mathrm{C}_{5}-\mathrm{C}_{4}-\mathrm{N}_{15}\right)(-$ $\left.179.0236^{\circ}\right), \quad\left(\mathrm{C}_{7} \mathrm{~B}-\mathrm{C}_{8} \mathrm{~B}-\mathrm{C}_{9} \mathrm{~B}-\mathrm{N}_{1} \mathrm{~B}\right) \quad\left(-178.5 \quad(7)^{\circ}\right)$ as displayed in Table 1. From the previous results it was noticed that the B3LYP/6-311G (d) more compatible with the confirmed structure and there are lowest difference between them (Farag and Fahim, 2019; Fahim and Shalaby, 2019; Dacrory and Fahim, 2020). 
Asmaa Aboelnaga et al. / OnLine Journal of Biological Sciences 2020, 20 (4): 207.220 DOI: 10.3844/ojbsci.2020.207.220

Table 1: Designated optimized bond length $\AA$ and bond angle degrees dihedral angle degrees of Compound CQT (3) X-ray of 4,7-dichloroquinoline and CQP(4) using B3LYP/6-311G(d)

Bond Length $\AA \quad$ Bond Angles $\left({ }^{\circ}\right)$

\begin{tabular}{|c|c|c|c|c|c|c|}
\hline $\begin{array}{l}\text { Parameters } \\
\text { of Bond } \\
\text { lengths }\end{array}$ & $\begin{array}{l}\mathrm{HF} / 6- \\
311 \mathrm{G}(\mathrm{d})\end{array}$ & $\begin{array}{l}\text { DFT/ } \\
\text { B3LYP/ } \\
6-311 G(d)\end{array}$ & \multicolumn{2}{|c|}{$\begin{array}{l}\text { X-ray } \\
\text { (Fahim and Shalaby, 2019) }\end{array}$} & $\begin{array}{l}\text { Parameters } \\
\text { of Bond } \\
\text { angles }\end{array}$ & $\mathrm{HF}$ \\
\hline Compound & $\mathrm{QT}(3)$ & & & & & \\
\hline $\mathrm{C}_{1}-\mathrm{C}_{2}$ & 1.29280 & 1.37280 & $\mathrm{C}_{11} \mathrm{~A}-\mathrm{C}_{4} \mathrm{~A}$ & $1.720(8)$ & $\mathrm{Cl}_{17}-\mathrm{C}_{6}-\mathrm{C}_{1}$ & 119.00273 \\
\hline $\mathrm{C}_{2}-\mathrm{C}_{3}$ & 1.33201 & 1.42201 & $\mathrm{C}_{12} \mathrm{~A}-\mathrm{C}_{7} \mathrm{~A}$ & $1.742(8)$ & $\mathrm{H}_{7}-\mathrm{C}_{1}-\mathrm{C}_{2}$ & 120.64767 \\
\hline $\mathrm{C}_{3}-\mathrm{C}_{4}$ & 1.42172 & 1.3896 & $\mathrm{~N}_{1} \mathrm{~A}-\mathrm{C}_{2} \mathrm{~A}$ & $1.328(11)$ & $\mathrm{H}_{8}-\mathrm{C}_{2}-\mathrm{C}_{3}$ & 118.60685 \\
\hline $\mathrm{C}_{4}-\mathrm{C}_{5}$ & 1.37334 & 1.37625 & $\mathrm{~N}_{1} \mathrm{~A}-\mathrm{C}_{9} \mathrm{~A}$ & $1.379(10)$ & $\mathrm{C}_{2}-\mathrm{C}_{3}-\mathrm{C}_{4}$ & 119.09588 \\
\hline $\mathrm{C}_{5}-\mathrm{C}_{6}$ & 1.76000 & 1.4023 & $\mathrm{C}_{2} \mathrm{~A}-\mathrm{C}_{3} \mathrm{~A}$ & $1.432(12)$ & $\mathrm{C}_{4}-\mathrm{C}_{5}-\mathrm{C}_{6}$ & 120.45593 \\
\hline $\mathrm{C}_{6}-\mathrm{Cl}_{17}$ & 1.10021 & 1.07856 & $\mathrm{C}_{2} \mathrm{~A}-\mathrm{H}_{2} \mathrm{AA}$ & 0.9500 & $\mathrm{Cl}_{17}-\mathrm{C}_{6}-\mathrm{C}_{5}$ & 120.64566 \\
\hline $\mathrm{C}_{5}-\mathrm{H}_{10}$ & 1.10033 & 1.2564 & $\mathrm{C}_{3} \mathrm{~A}-\mathrm{C}_{4} \mathrm{~A}$ & $1.362(11)$ & $\mathrm{C}_{5}-\mathrm{C}_{4}-\mathrm{C}_{3}$ & 119.21034 \\
\hline $\mathrm{C}_{2}-\mathrm{H}_{8}$ & 1.42203 & 1.02365 & $\mathrm{C}_{3} \mathrm{~A}-\mathrm{H}_{3} \mathrm{AA}$ & 0.9500 & $\mathrm{C}_{5}-\mathrm{C}_{4}-\mathrm{C}_{3}$ & 121.73001 \\
\hline $\mathrm{C}_{3}-\mathrm{C}_{9}$ & 1.42208 & 1.389523 & $\mathrm{C}_{4} \mathrm{~A}-\mathrm{C}_{10} \mathrm{~A}$ & $1.422(11)$ & $\mathrm{C}_{5}-\mathrm{C}_{4}-\mathrm{N}_{16}$ & 120.54903 \\
\hline $\mathrm{C}_{4}-\mathrm{N}_{16}$ & 1.37286 & 1.30256 & $\mathrm{C}_{5} \mathrm{~A}-\mathrm{C}_{6} \mathrm{~A}$ & $1.348(12)$ & $\mathrm{C}_{4}-\mathrm{N}_{16}-\mathrm{C}_{11}$ & 120.36687 \\
\hline $\mathrm{N}_{16}-\mathrm{C}_{11}$ & 1.37286 & 1.30236 & $\mathrm{C}_{5} \mathrm{~A}-\mathrm{C}_{10} \mathrm{~A}$ & $1.420(11)$ & $\mathrm{N}_{16}-\mathrm{C}_{11}-\mathrm{C}_{12}$ & 120.86227 \\
\hline $\mathrm{C}_{11}-\mathrm{C}_{12}$ & 1.41623 & 1.23012 & $\mathrm{C}_{5} \mathrm{~A}-\mathrm{H}_{5} \mathrm{AA}$ & 0.9500 & $\mathrm{C}_{12}-\mathrm{C}_{9}-\mathrm{N}_{18}$ & 109.47 \\
\hline $\mathrm{C}_{12}-\mathrm{C}_{9}$ & 1.37294 & 1.39853 & $\mathrm{C}_{6} \mathrm{~A}-\mathrm{C}_{7} \mathrm{~A}$ & 1.417 (12) & $\mathrm{N}_{18}-\mathrm{N}_{19}-\mathrm{H}_{21}$ & 119.88054 \\
\hline $\mathrm{C}_{9}-\mathrm{N}_{18}$ & 1.47000 & 1.0325 & $\mathrm{C}_{6} \mathrm{~A}-\mathrm{H}_{6} \mathrm{AA}$ & 0.9500 & $\mathrm{~S}_{23}-\mathrm{C}_{22}-\mathrm{N}_{24}$ & 109.47117 \\
\hline $\mathrm{N}_{18}-\mathrm{N}_{19}$ & 1.4000 & 1.30261 & $\mathrm{C}_{7} \mathrm{~A}-\mathrm{C}_{8} \mathrm{~A}$ & $1.364(11)$ & $\mathrm{H}_{25}-\mathrm{N}_{24}-\mathrm{C}_{22}$ & 41.98580 \\
\hline $\mathrm{N}_{19}-\mathrm{H}_{21}$ & 1.0000 & 1.39652 & $\mathrm{C}_{8}-\mathrm{C}_{9} \mathrm{~A}$ & $1.421(11)$ & $\mathrm{N}_{18}-\mathrm{N}_{19}-\mathrm{H}_{21}$ & \\
\hline $\mathrm{C}_{27}-\mathrm{N}_{24}$ & 1.47000 & 1.20325 & $\mathrm{C}_{8}-\mathrm{H}_{8} \mathrm{AA}$ & 0.9500 & & \\
\hline $\mathrm{N}_{24}-\mathrm{H}_{25}$ & 1.0000 & 1.2351 & $\mathrm{C}_{9} \mathrm{~A}-\mathrm{C}_{10} \mathrm{~A}$ & $1.422(11)$ & & \\
\hline $\mathrm{C}_{22}-\mathrm{S}_{23}$ & 1.56640 & 1.56640 & & & & \\
\hline
\end{tabular}

DFT/

B3LYP/

6-311G(d) X-ray (Fahim and Shalaby, 2019)

118.2311 $\mathrm{C}_{2} \mathrm{~A}-\mathrm{N}_{1} \mathrm{~A}-\mathrm{C}_{9} \mathrm{~A} \quad 117.2(8)$

$121.5232 \quad \mathrm{~N}_{1} \mathrm{~A}-\mathrm{C}_{2} \mathrm{~A}-\mathrm{C}_{3} \mathrm{~A} \quad 124.2(7)$

$116.2580 \quad \mathrm{~N}_{1} \mathrm{~A}-\mathrm{C}_{2} \mathrm{~A}-\mathrm{H}_{2} \mathrm{AA} \quad 117.9$

$\begin{array}{lll}119.5232 & \mathrm{C}_{5} \mathrm{~A}-\mathrm{C}_{10} \mathrm{~A}-\mathrm{C}_{9} \mathrm{~A} & 119.3(7)\end{array}$

$116.9875 \quad \mathrm{C}_{4} \mathrm{~A}-\mathrm{C}_{3} \mathrm{~A}-\mathrm{C}_{2} \mathrm{~A} \quad 117.7(7)$

$122.0236 \quad \mathrm{C}_{4} \mathrm{~A}-\mathrm{C}_{3} \mathrm{~A}-\mathrm{H} 3 \mathrm{AA} \quad 121.1$

$\begin{array}{lll}117.0236 & \text { C4A-C10A-C9A } & 116.4(7)\end{array}$

121.5230 C3A-C4A-C10A $121.2(8)$

$120.0251 \quad$ C3A-C4A-Cl1A $\quad 119.5(7)$

119.8651 C10A-C4A-Cl1A $119.4(6)$

$\begin{array}{lll}120.5236 & \text { C6A-C5A-C10A } 120.2(8)\end{array}$

$116.325 \quad \mathrm{C}_{6} \mathrm{~A}-\mathrm{C}_{5} \mathrm{~A}-\mathrm{H}_{5} \mathrm{AA} \quad 119.9$

$120.5236 \quad \mathrm{C}_{4} \mathrm{~A}-\mathrm{C}_{10} \mathrm{~A}-\mathrm{C}_{5} \mathrm{~A}(8) \quad 124.2(8)$

$\begin{array}{lll}115.4562 & \mathrm{C}_{5} \mathrm{~A}-\mathrm{C}_{6} \mathrm{~A}-\mathrm{C}_{7} \mathrm{~A} & 120.5(7)\end{array}$

109.2365 $\mathrm{C}_{5} \mathrm{~A}-\mathrm{C}_{6} \mathrm{~A}-\mathrm{H}_{6} \mathrm{AA} \quad 119.8$

C8A-C 9 A- $\mathrm{C}_{10} \mathrm{~A} \quad 119.5(7)$

$\mathrm{C}_{8} \mathrm{~A}-\mathrm{C}_{7} \mathrm{~A}-\mathrm{C}_{6} \mathrm{~A} \quad 121.7(8)$

C8A-C7A-C $\mathrm{C}_{12} \mathrm{~A} \quad 119.7(7)$

$\mathrm{C}_{6} \mathrm{~A}-\mathrm{C}_{7} \mathrm{~A}-\mathrm{C}_{12} \mathrm{~A} \quad 118.6(6)$

$\mathrm{C}_{7} \mathrm{~A}-\mathrm{C}_{8} \mathrm{~A}-\mathrm{C}_{9} \mathrm{~A} \quad 118.9(8)$

$\mathrm{C}_{7} \mathrm{~A}-\mathrm{C}_{8} \mathrm{~A}-\mathrm{H}_{8} \mathrm{AA} \quad 120.6$

$\mathrm{N}_{1} \mathrm{~A}-\mathrm{C}_{9} \mathrm{~A}-\mathrm{C}_{8} \mathrm{~A} \quad 117.3(8)$

$\mathrm{N}_{1} \mathrm{~A}-\mathrm{C}_{9} \mathrm{~A}-\mathrm{C}_{10} \mathrm{~A}$

Dihedral angles $\left({ }^{\circ}\right)$

$\mathrm{H}_{20}-\mathrm{N}_{19}-\mathrm{N}_{18}-\mathrm{C}_{22}-109.7568$

$\mathrm{N}_{19}-\mathrm{N}_{18}-\mathrm{C}_{22}-\mathrm{S}_{23} \quad 126.7877$

$\mathrm{H}_{25}-\mathrm{N}_{24}-\mathrm{C}_{22}-\mathrm{S}_{23} \quad-63.32708$

$\mathrm{H}_{25}-\mathrm{N}_{24}-\mathrm{C}_{22}-\mathrm{N}_{18} \quad 116.67290$

$\mathrm{Cl}_{17}-\mathrm{C}_{6}-\mathrm{C}_{1}-\mathrm{C}_{2} \quad-179.9936$

$\mathrm{N}_{16}-\mathrm{C}_{4}-\mathrm{C}_{3}-\mathrm{C}_{9} \quad-0.00075$

Compound CQP(4)

$\mathrm{N}_{17-\mathrm{N}_{25}} \quad 1.4700$

$\mathrm{N}_{25}-\mathrm{C}_{19} \quad 1.39516$

1.5912

1.4852

$\mathrm{C}_{11} \mathrm{~B}-\mathrm{C}_{4} \mathrm{~B}$

$1.769(8)$

$\begin{array}{ll}\mathrm{N}_{1} \mathrm{~B}-\mathrm{C}_{2} \mathrm{~B} & 1.313(11) \\ \mathrm{N}_{1} \mathrm{~B}-\mathrm{C}_{9} \mathrm{~B} & 1.368(10)\end{array}$

1.3526

$\mathrm{C}_{19}-\mathrm{N}_{27} \quad 1.3947$

$\mathrm{N}_{27}-\mathrm{C}_{22} \quad 1.39543$

$\mathrm{N}_{27}-\mathrm{H}_{23} \quad 1.09968$

1.4256

1.4123

1.16595

$\mathrm{C}_{22}-\mathrm{C}_{28} \quad 1.54000 \quad 1.48852$

$\mathrm{C}_{28}-\mathrm{H}_{29} \quad 1.07000 \quad 0.9965$

$\mathrm{C}_{4}-\mathrm{N}_{15} \quad 1.42208 \quad 1.5032$

$\begin{array}{lll}\mathrm{C}_{22}-\mathrm{C}_{21} & 1.39482 & 1.3865\end{array}$

$\begin{array}{lll}\mathrm{C}_{21}-\mathrm{H}_{24} & 1.09976 & 1.3265\end{array}$

$\mathrm{C}_{21}-\mathrm{C}_{20} \quad 1.39514$

$\mathrm{C}_{20}-\mathrm{O}_{32} \quad 1.25840$

$\mathrm{C}_{17}-\mathrm{C}_{9} \quad 1.47000$

$\mathrm{C}_{9}-\mathrm{C}_{3} \quad 1.42203$

$\mathrm{C}_{3}-\mathrm{C}_{4} \quad 1.41887$

$\mathrm{C}_{4}-\mathrm{N}_{15} \quad 1.42208$

$\mathrm{C}_{11}-\mathrm{C}_{12} \quad 1.41622$

$\mathrm{N}_{15}-\mathrm{C}_{11} \quad 1.37289$

$\mathrm{C}_{4}-\mathrm{C}_{5} \quad 1.42172$

$\mathrm{C}_{5}-\mathrm{C}_{6} \quad 1.37334$

$\mathrm{C}_{2} \mathrm{~B}-\mathrm{C}_{3} \mathrm{~B} \quad 1.418(11)$

$\mathrm{C}_{2} \mathrm{~B}-\mathrm{H}_{2} \mathrm{BA} \quad 0.9500$

$\mathrm{C}_{3} \mathrm{~B}-\mathrm{C}_{4} \mathrm{~B} \quad 1.338(12)$

$\mathrm{C}_{3} \mathrm{~B}-\mathrm{H}_{3} \mathrm{BA} \quad 0.9500$

$\mathrm{C}_{4} \mathrm{~B}-\mathrm{C}_{10} \mathrm{~B} \quad 1.443(11)$

$\mathrm{C}_{5} \mathrm{~B}-\mathrm{C}_{6} \mathrm{~B} \quad 1.373(12)$

$\mathrm{C}_{5} \mathrm{~B}-\mathrm{C}_{10} \mathrm{~B} \quad 1.407(12)$

$\mathrm{C}_{5} \mathrm{~B}-\mathrm{H}_{5} \mathrm{BA} \quad 0.9500$

0.96598
1.238652

1.3652

1.3256

1.39652

1.4236

1.4235

1.33256

1.42172

$\mathrm{C}_{6}-\mathrm{Cl}_{16} \quad 1.7600$

$\mathrm{C}_{6} \mathrm{~B}-\mathrm{C}_{7} \mathrm{~B} \quad 1.410(11)$

$\mathrm{C}_{6} \mathrm{~B}-\mathrm{H}_{6} \mathrm{BA} \quad 0.9500$

$\mathrm{C}_{7} \mathrm{~B}-\mathrm{C}_{8} \mathrm{~B} \quad 1.359(12)$

$\mathrm{C}_{8} \mathrm{~B}-\mathrm{C}_{9} \mathrm{~B} \quad 1.400(11)$

$\mathrm{C}_{8} \mathrm{~B}-\mathrm{H}_{8} \mathrm{BA} \quad 0.9500$

$\mathrm{C}_{9} \mathrm{~B}-\mathrm{C}_{10} \mathrm{~B} \quad 1.429(10)$

$\mathrm{S}_{26}-\mathrm{C}_{19}-\mathrm{N} 27 \quad 120.01052$

$\mathrm{C}_{19}-\mathrm{N}_{27}-\mathrm{H}_{23} \quad 120.01279$

$\mathrm{C}_{19}-\mathrm{N}_{27}-\mathrm{C}_{22} \quad 119.99412$

$\mathrm{N}_{27}-\mathrm{C}_{22}-\mathrm{C}_{28} \quad 119.98110$

$\mathrm{C}_{22}-\mathrm{C}_{21}-\mathrm{C}_{20} \quad 120.00469$

$\mathrm{C}_{21}-\mathrm{C}_{20}-\mathrm{O}_{32} \quad 119.99197$

$\mathrm{O}_{32}-\mathrm{C}_{20}-\mathrm{N}_{25} \quad 120.00800$

$\mathrm{C}_{20}-\mathrm{N}_{25}-\mathrm{N}_{17} \quad 120.00430$

$\mathrm{N}_{17}-\mathrm{C}_{9}-\mathrm{C}_{12} \quad 120.86230$

$\mathrm{C}_{12}-\mathrm{C}_{11}-\mathrm{N}_{15} \quad 120.36689$

$\mathrm{N}_{15}-\mathrm{C}_{4}-\mathrm{C}_{3} \quad 119.0596$

$\mathrm{C}_{4}-\mathrm{C}_{5}-\mathrm{C}_{6} \quad 120.4558$

$\mathrm{C}_{5}-\mathrm{C}_{6}-\mathrm{Cl}_{16} \quad 120.65564$

$-108.235$

128.2365

127.232

110.231

$-179.5231$

$-0.00072$

121.2356

123.2562

116.982

119.2365

120.523

119.869

121.5965

121.5621

120.235

122.5695

119.2356

120.2565

120.0236

$\mathrm{C}_{9} \mathrm{~A}-\mathrm{N}_{1} \mathrm{~A}-\mathrm{C}_{2} \mathrm{~A}-\mathrm{C}_{3} \mathrm{~A} \quad 0.9(12)$

C6A-C5A-C ${ }_{10} \mathrm{~A}-\mathrm{C}_{4} \mathrm{~A} \quad 178.6$ (7)

$\mathrm{C}_{2} \mathrm{~A}-\mathrm{N}_{1} \mathrm{~A}-\mathrm{C}_{9} \mathrm{~A}-\mathrm{C}_{8} \mathrm{~A} \quad 179.4$ (7)

$\mathrm{C}_{7} \mathrm{~A}-\mathrm{C}_{8} \mathrm{~A}-\mathrm{C}_{9} \mathrm{~A}-\mathrm{N}_{1} \mathrm{~A} \quad-178.8(7)$

$\mathrm{C}_{3} \mathrm{~A}-\mathrm{C}_{4} \mathrm{~A}-\mathrm{C}_{10} \mathrm{~A}-\mathrm{C}_{5} \mathrm{~A} \quad-179.7$ (7)

$\mathrm{C}_{3} \mathrm{~A}-\mathrm{C}_{4} \mathrm{~A}-\mathrm{C}_{10} \mathrm{~A}-\mathrm{C}_{9} \mathrm{~A} \quad-1.1(11)$

$\mathrm{C}_{2} \mathrm{~B}-\mathrm{N}_{1} \mathrm{~B}-\mathrm{C}_{9} \mathrm{~B}$

$116.3(7)$

$\mathrm{N}_{1} \mathrm{~B}-\mathrm{C}_{2} \mathrm{~B}-\mathrm{C}_{3} \mathrm{~B} \quad 124.9(8)$

$\mathrm{N}_{1} \mathrm{~B}-\mathrm{C}_{2} \mathrm{~B}-\mathrm{H}_{2} \mathrm{BA} \quad 117.6$

$\mathrm{C}_{4} \mathrm{~B}-\mathrm{C}_{3} \mathrm{~B}-\mathrm{C}_{2} \mathrm{~B} \quad 118.8$ (8)

$\mathrm{C}_{4} \mathrm{~B}-\mathrm{C}_{3} \mathrm{~B}-\mathrm{H}_{3} \mathrm{BA} \quad 120.6$

$\mathrm{C}_{3} \mathrm{~B}-\mathrm{C}_{4} \mathrm{~B}-\mathrm{C}_{10} \mathrm{~B} \quad 120.7$ (7)

$\mathrm{C}_{3} \mathrm{~B}-\mathrm{C}_{4} \mathrm{~B}-\mathrm{Cl}_{1} \mathrm{~B} \quad 121.4(6)$

$\mathrm{C}_{10} \mathrm{~B}-\mathrm{C}_{4} \mathrm{~B}-\mathrm{Cl}_{1} \mathrm{~B} \quad 117.9(6)$

$\mathrm{C}_{6} \mathrm{~B}-\mathrm{C}_{5} \mathrm{~B}-\mathrm{C}_{10} \mathrm{~B} \quad 121.7$ (7)

$\mathrm{C}_{6} \mathrm{~B}-\mathrm{C}_{5} \mathrm{~B}-\mathrm{H}_{5} \mathrm{BA} \quad 119.1$

$\mathrm{C}_{5} \mathrm{~B}-\mathrm{C}_{6} \mathrm{~B}-\mathrm{C}_{7} \mathrm{~B} \quad 117.0(8)$

$\mathrm{C}_{5} \mathrm{~B}-\mathrm{C}_{6} \mathrm{~B}-\mathrm{H}_{6} \mathrm{BA}$

$\mathrm{C}_{8} \mathrm{~B}-\mathrm{C}_{7} \mathrm{~B}-\mathrm{C}_{6} \mathrm{~B} \quad 123.7(7)$

$\mathrm{C}_{8} \mathrm{~B}-\mathrm{C}_{7} \mathrm{~B}-\mathrm{C}_{12} \mathrm{~B} \quad 119.8(6)$

$\mathrm{C}_{6} \mathrm{~B}-\mathrm{C}_{7} \mathrm{~B}-\mathrm{C}_{12} \mathrm{~B} \quad 116.5(7)$

$\mathrm{C}_{7} \mathrm{~B}-\mathrm{C}_{8} \mathrm{~B}-\mathrm{C} 9 \mathrm{~B} \quad 119.4(7)$

$\mathrm{C}_{7} \mathrm{~B}-\mathrm{C}_{8} \mathrm{~B}-\mathrm{H}_{8} \mathrm{BA} \quad 120.3$

$\mathrm{N}_{1} \mathrm{~B}-\mathrm{C}_{9} \mathrm{~B}-\mathrm{C}_{8} \mathrm{~B} \quad 117.0(7)$

$\mathrm{N}_{1} \mathrm{~B}-\mathrm{C}_{9} \mathrm{~B}-\mathrm{C}_{10} \mathrm{~B} \quad 124.4(7)$

$\mathrm{C}_{8} \mathrm{~B}-\mathrm{C}_{9} \mathrm{~B}-\mathrm{C}_{10} \mathrm{~B} \quad 118.7(7)$

$\mathrm{C}_{5} \mathrm{~B}-\mathrm{C}_{10} \mathrm{~B}-\mathrm{C}_{9} \mathrm{~B} \quad 119.3(7)$

$\mathrm{C}_{5} \mathrm{~B}-\mathrm{C}_{10} \mathrm{~B}-\mathrm{C}_{4} \mathrm{~B}$

$\mathrm{C}_{9} \mathrm{~B}-\mathrm{C}_{10} \mathrm{~B}-\mathrm{C}_{4} \mathrm{~B} \quad 114.9(7)$

Dihedral angles $\left(^{\circ}\right)$

$\mathrm{N}_{27}-\mathrm{C}_{22}-\mathrm{C}_{28}-\mathrm{H}_{29} \quad-59.11679$

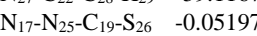

$\mathrm{N}_{17}-\mathrm{N}_{25}-\mathrm{C}_{19}-\mathrm{N}_{27}-179.97295$

$\mathrm{C}_{9}-\mathrm{N}_{17}-\mathrm{N}_{25}-\mathrm{C}_{20} \quad-90.85212$

$\mathrm{O}_{32}-\mathrm{C}_{20}-\mathrm{N}_{25}-\mathrm{N}_{17}-0.00554$

$\mathrm{C}_{6}-\mathrm{C}_{5}-\mathrm{C}_{4}-\mathrm{N}_{15} \quad 179.99158$

$-80.3256 \quad \mathrm{C}_{9} \mathrm{~B}-\mathrm{N}_{1} \mathrm{~B}-\mathrm{C}_{2} \mathrm{~B}-\mathrm{C}_{3} \mathrm{~B} \quad 1.5(12)$

$-0.02365 \quad \mathrm{C}_{2} \mathrm{~B}-\mathrm{C}_{3} \mathrm{~B}-\mathrm{C}_{4} \mathrm{~B}-\mathrm{Cl}_{1} \mathrm{~B} \quad-179.5(6)$

$-179.2356 \quad \mathrm{C}_{5} \mathrm{~B}-\mathrm{C}_{6} \mathrm{~B}-\mathrm{C}_{7} \mathrm{~B}-\mathrm{C}_{12} \mathrm{~B} \quad 179.9(6)$

$-88.3625 \quad \mathrm{C}_{2} \mathrm{~B}-\mathrm{N}_{1} \mathrm{~B}-\mathrm{C}_{9} \mathrm{~B}-\mathrm{C}_{8} \mathrm{~B} \quad 178.4$ (7)

$-0.00256 \quad \mathrm{C}_{7} \mathrm{~B}-\mathrm{C}_{8} \mathrm{~B}-\mathrm{C}_{9} \mathrm{~B}-\mathrm{N}_{1} \mathrm{~B}-\quad-178.5(7)$

$-179.0236$

\section{Physical Characterization}

Moreover, the physical calculation boundaries of compound CQT (3) and CQP (4), like, absolute electronegativities $(\chi)$, global Softness $(\mathrm{S})$, absolute hardness, $(\eta)$ absolute softness $(\sigma)$, chemical potentials $(P i)$, global electrophilicity $(\omega)$ and additional electronic charge, $\left(\Delta N_{\max }\right)$, were tabulated in Table 2 according to the Equations (2-8) and DFT/B3LYP/6-31G(d) used to optimize it (Dacrory and Fahim, 2020). The molecular 
structure of these compounds was not planar. The potential activities presented in the precursor compounds CQP (4) due to presence of tetrahydro-6-methyl-2thioxopyrimidin-4(1H)-one which increased the activity and more stability rather than CQT (3) due to resonance of electrons and stability of ring attached to chloroquinone, Another point of view, The $\pi$ isoelectronic structures of compound CQT (3) and CQP (4) utilizing DFT/B3LYP/6-311G(d) and HF/6-311G(d) basis set, whereas the difference in CQT between two basis set $(160 \mathrm{eV})(\approx 3800 \mathrm{kcal} / \mathrm{mol})$, but the CQP (4) difference was $(182.126 \mathrm{eV})(\approx 4199.92 \mathrm{kcal} / \mathrm{mol})$ which these difference indicate the stability of CQP (4). Moreover, the dipole moment $(\mu)$ difference for HF and B3LYP/6-311G(d) basis sets for CQT (3) through (2.9217D) and CQP (4) was (1.3353D) which indicate that the easily charge separation of CQP(4) (Atkins and De Paula, 2011) as shown in Table 2:

$$
\begin{aligned}
& \Delta E=E_{\text {LUMO }}-E_{\text {Номо }} \\
& \chi=\frac{-\left(E_{\text {LUMO }}+E_{\text {HОMO }}\right)}{2} \\
& \eta=\frac{\left(E_{\text {LUMO }}-E_{\text {HомO }}\right)}{2} \\
& \sigma=1 / \eta
\end{aligned}
$$

$$
\begin{aligned}
& P i=-X \\
& S=1 / 2 \eta \\
& \omega=P i^{2} / 2 \eta \\
& \Delta N_{\max }=-P i / \eta
\end{aligned}
$$

The absolute electronegativities $(\chi)$ which concept that designates the affinity of an atom to attract a mutual pair of electrons, the value of $(\chi)$ for CQT (3) was $(5.798 \mathrm{eV})(\approx 133.71286 \mathrm{kcal} / \mathrm{mol})$ and CQP (4) was $(1.1574351 \mathrm{eV}) \quad(\approx 26.69107 \mathrm{kcal} / \mathrm{mol})$, which confirm that the higher value of thiosemicarbazide CQT (3) which attract the atoms of ethylacetoacetate to form the tetrahydro-6-methyl-2-thioxopyrimidin4(1H)-one (Yamamoto et al., 1998).

The absolute hardness $(\eta)$ which measure of the resistance to change in electron density around the molecule, CQT (3) difference between HF/6-311G(d) and DFT/B3LYP/6-31G(d) (2.1739519 eV) $(\approx 50.13251 \mathrm{kcal} / \mathrm{mol})$, also the CQP (4) different $(3.1191 \mathrm{eV}) \quad(\approx 71.9258 \mathrm{kcal} / \mathrm{mol})$ which the more electron density of CQP and stability of this

\begin{tabular}{|c|c|c|c|c|c|c|c|c|c|}
\hline \multicolumn{5}{|c|}{ DFT/B3LYP/6-311G(d) } & \multicolumn{5}{|l|}{$\mathrm{HF} / 6-311 \mathrm{G}(\mathrm{d})$} \\
\hline \multicolumn{4}{|c|}{ Compound CQT } & \multirow{2}{*}{$\begin{array}{l}\text { Compound CQP } \\
-1692.634\end{array}$} & \multirow{2}{*}{$\begin{array}{l}\text { Compound CQT } \\
\mathrm{E}_{\mathrm{T}}(\mathrm{au})\end{array}$} & \multicolumn{4}{|c|}{ Compound CQP } \\
\hline$E_{\mathrm{T}}(\mathrm{au})$ & \multicolumn{3}{|c|}{-1464.3216} & & & \multicolumn{2}{|c|}{-1458.83632} & \multicolumn{2}{|c|}{-1685.9406} \\
\hline$E_{\text {Номо }}(\mathrm{au})$ & \multicolumn{3}{|c|}{-0.16212} & -0.24111 & $\mathrm{E}_{\text {Номо }}(\mathrm{au})$ & \multicolumn{2}{|c|}{-0.45510} & \multicolumn{2}{|c|}{-0.31274} \\
\hline$E_{\text {LUMO }}(\mathrm{au})$ & \multicolumn{3}{|c|}{-0.10736} & -0.10238 & $\mathrm{E}_{\text {LUMO }}(\mathrm{au})$ & \multicolumn{2}{|c|}{-0.24055} & \multicolumn{2}{|c|}{0.05432} \\
\hline $\mathrm{Eg}(\mathrm{eV})$ & \multicolumn{3}{|c|}{1.4900962} & 3.7750374 & $\mathrm{Eg}(\mathrm{eV})$ & \multicolumn{2}{|c|}{5.8382057} & \multicolumn{2}{|c|}{18.24796} \\
\hline$\mu(D)$ & \multicolumn{3}{|c|}{4.6871} & 8.9694 & $\mu(D)$ & \multicolumn{2}{|c|}{7.6088} & \multicolumn{2}{|c|}{10.3047} \\
\hline$\chi(\mathrm{eV})$ & \multicolumn{3}{|c|}{3.6664639} & 4.67342172 & $\chi(\mathrm{eV})$ & \multicolumn{2}{|c|}{9.46480486} & \multicolumn{2}{|c|}{3.5159849} \\
\hline$\eta(\mathrm{eV})$ & \multicolumn{3}{|c|}{0.7450481} & 1.88751869 & $\eta(\mathrm{eV})$ & \multicolumn{2}{|c|}{2.91910283} & \multicolumn{2}{|c|}{4.9941081} \\
\hline$\sigma(\mathrm{eV})$ & \multicolumn{3}{|c|}{993.8419} & 392.2795 & $\sigma(\mathrm{eV})$ & \multicolumn{2}{|c|}{253.6602097} & \multicolumn{2}{|c|}{148.26675} \\
\hline $\mathrm{P}_{\mathrm{i}}(\mathrm{eV})$ & \multicolumn{3}{|c|}{-3.6664639} & -4.67342172 & $\mathrm{P}_{\mathrm{i}}(\mathrm{eV})$ & \multicolumn{2}{|c|}{-9.464804857} & \multicolumn{2}{|c|}{-3.5159849} \\
\hline $\mathrm{S}(\mathrm{eV})$ & 0.372 & & & 0.94369132 & $\mathrm{~S}(\mathrm{eV})$ & 1.45 & & 2.49 & \\
\hline$\omega(\mathrm{eV})$ & 0.067 & & & 0.027837261177 & $\omega(\mathrm{eV})$ & 0.01 & & 0.04 & \\
\hline$\Delta \mathrm{N}_{\max }$ & 133. & & & 67.3727 & $\Delta \mathrm{N}_{\max }$ & 88.2 & & 19.1 & \\
\hline Net charges & $\mathrm{N}_{18}$ & -0.384 & $\mathrm{~N}_{17}$ & -0.465 & Net charges & $\mathrm{N}_{18}$ & -0.510 & $\mathrm{~N}_{17}$ & -0.544 \\
\hline & $\mathrm{N}_{19}$ & -0.550 & $\mathrm{H}_{18}$ & 0.352 & & $\mathrm{~N}_{19}$ & -0.605 & $\mathrm{H}_{18}$ & 0.380 \\
\hline & $\mathrm{H}_{21}$ & 0.295 & $\mathrm{~N}_{25}$ & -0.497 & & $\mathrm{H}_{21}$ & 0.335 & $\mathrm{~N}_{25}$ & -0.680 \\
\hline & $\mathrm{C}_{22}$ & -0.119 & $\mathrm{C}_{19}$ & 0.124 & & $\mathrm{C}_{22}$ & -0.004 & $\mathrm{C}_{19}$ & 0.320 \\
\hline & $\mathrm{H}_{20}$ & 0.330 & $\mathrm{~S}_{26}$ & -0.018 & & $\mathrm{H}_{20}$ & 0.377 & $\mathrm{~S}_{26}$ & -0.084 \\
\hline & $\mathrm{S}_{23}$ & 0.056 & $\mathrm{~N}_{27}$ & -0.681 & & $\mathrm{~S}_{23}$ & 0.186 & $\mathrm{~N}_{27}$ & -0.890 \\
\hline & $\mathrm{N}_{24}$ & -0.679 & $\mathrm{H}_{23}$ & 0.398 & & $\mathrm{~N}_{24}$ & -0.812 & $\mathrm{H}_{23}$ & 0.449 \\
\hline & & & $\mathrm{C}_{22}$ & 0.390 & & & & $\mathrm{C}_{22}$ & 0.509 \\
\hline & ${ }^{\mathrm{a}} \mathrm{Eg}=$ & $E_{\text {НОмо. }}$ & & & & & & & \\
\hline
\end{tabular}
compound (Yang and Parr, 1985).

Absolute softness $(\sigma)$ indicate the interaction of the compound, the difference of CQT (3) $(740.1817 \mathrm{eV})$ while CQP (4) was (244.0135 eV), the large difference for CQT indicate that the activity of CQT to react with ethylacetoacetate (Fahim et al., 2020).

Table 2: Ground state energies of compounds CQT (3) and CQP (4) utilizing DFT/B3LYP/6-311G(d) and HF/6-31G(d)and their physical parameters 
Table 3: The calculated Mullikan and NBO charges of CQT (3) and CQP (4) utilizing DFT/ B3LYP/6-311G(d) DFT/ B3LYP/6-311G(d)

\begin{tabular}{|c|c|c|c|c|c|c|c|}
\hline \multicolumn{4}{|c|}{ Total Mullikan charges } & \multicolumn{4}{|c|}{ NBO Charges } \\
\hline \multicolumn{2}{|c|}{ Compound CQT } & \multicolumn{2}{|c|}{ Compound CQP } & \multicolumn{2}{|c|}{ Compound CQT } & \multicolumn{2}{|c|}{ Compound CQP } \\
\hline $\mathrm{C}_{1}$ & -0.073 & $\mathrm{C}_{1}$ & 0.079 & $\mathrm{C}_{1}$ & -0.228 & $\mathrm{C}_{1}$ & -0.221 \\
\hline $\mathrm{C}_{2}$ & -0.062 & $\mathrm{C}_{2}$ & -0.005 & $\mathrm{C}_{2}$ & -0.186 & $\mathrm{C}_{2}$ & -0.146 \\
\hline $\mathrm{C}_{3}$ & -0.191 & $\mathrm{C}_{3}$ & -0.072 & $\mathrm{C}_{3}$ & -0.087 & $\mathrm{C}_{3}$ & -0.094 \\
\hline $\mathrm{C}_{4}$ & -0.434 & $\mathrm{C}_{4}$ & 0.116 & $\mathrm{C}_{4}$ & 0.202 & $\mathrm{C}_{4}$ & 0.177 \\
\hline $\mathrm{C}_{5}$ & -0.071 & $\mathrm{C}_{5}$ & -0.011 & $\mathrm{C}_{5}$ & -0.277 & $\mathrm{C}_{5}$ & -0.278 \\
\hline $\mathrm{C}_{6}$ & -0.308 & $\mathrm{C}_{6}$ & -0.294 & $\mathrm{C}_{6}$ & -0.031 & $\mathrm{C}_{6}$ & -0.018 \\
\hline $\mathrm{H}_{7}$ & 0.171 & $\mathrm{Cl}_{16}$ & 0.003 & $\mathrm{H}_{7}$ & 0.221 & $\mathrm{Cl}_{16}$ & -0.002 \\
\hline $\mathrm{H}_{8}$ & 0.176 & $\mathrm{H}_{10}$ & 0.189 & $\mathrm{H}_{8}$ & 0.232 & $\mathrm{H}_{10}$ & 0.240 \\
\hline $\mathrm{Cl}_{17}$ & -0.015 & $\mathrm{H}_{7}$ & 0.181 & $\mathrm{Cl}_{17}$ & -0.016 & $\mathrm{H}_{7}$ & 0.225 \\
\hline $\mathrm{H}_{10}$ & 0.173 & $\mathrm{H}_{8}$ & 0.195 & $\mathrm{H}_{10}$ & 0.217 & $\mathrm{H}_{8}$ & 0.195 \\
\hline $\mathrm{N}_{16}$ & -0.830 & $\mathrm{~N}_{15}$ & -0.422 & $\mathrm{~N}_{16}$ & -0.566 & $\mathrm{~N}_{15}$ & -0.438 \\
\hline $\mathrm{H}_{13}$ & 0.341 & $\mathrm{C}_{11}$ & 0.039 & $\mathrm{H}_{13}$ & 0.410 & $\mathrm{C}_{11}$ & 0.053 \\
\hline $\mathrm{C}_{11}$ & -0.124 & $\mathrm{C}_{12}$ & -0.168 & $\mathrm{C}_{11}$ & -0.005 & $\mathrm{C}_{12}$ & -0.267 \\
\hline $\mathrm{H}_{14}$ & 0.176 & $\mathrm{C}_{9}$ & 0.209 & $\mathrm{H}_{14}$ & 0.205 & $\mathrm{C}_{9}$ & 0.184 \\
\hline $\mathrm{C}_{11}$ & 0.124 & $\mathrm{H}_{13}$ & 0.176 & $\mathrm{C}_{11}$ & -0.005 & $\mathrm{H}_{13}$ & 0.201 \\
\hline $\mathrm{C}_{12}$ & -0.129 & $\mathrm{H}_{14}$ & 0.185 & $\mathrm{C}_{12}$ & -0.223 & $\mathrm{H}_{14}$ & 0.226 \\
\hline $\mathrm{H}_{15}$ & 0.131 & $\mathrm{C}_{12}$ & 0.168 & $\mathrm{H}_{15}$ & 0.217 & $\mathrm{C}_{12}$ & -0.267 \\
\hline $\mathrm{C}_{9}$ & 0.342 & $\mathrm{~N}_{17}$ & -0.465 & $\mathrm{C}_{9}$ & 0.148 & $\mathrm{~N}_{17}$ & -0.457 \\
\hline $\mathrm{N}_{18}$ & -0.384 & $\mathrm{H}_{18}$ & 0.352 & $\mathrm{~N}_{18}$ & -0.337 & $\mathrm{H}_{18}$ & 0.388 \\
\hline $\mathrm{N}_{19}$ & -0.550 & $\mathrm{~N}_{25}$ & -0.497 & $\mathrm{~N}_{19}$ & -0.614 & $\mathrm{~N}_{25}$ & -0.457 \\
\hline $\mathrm{H}_{21}$ & 0.295 & $\mathrm{C}_{19}$ & 0.124 & $\mathrm{H}_{21}$ & 0.332 & $\mathrm{C}_{19}$ & -0.270 \\
\hline $\mathrm{C}_{22}$ & -0.119 & $S_{26}$ & -0.018 & $\mathrm{C}_{22}$ & 0.158 & $\mathrm{~S}_{26}$ & 0.212 \\
\hline $\mathrm{H}_{20}$ & 0.330 & $\mathrm{~N}_{27}$ & -0.681 & $\mathrm{H}_{20}$ & 0.352 & $\mathrm{~N}_{27}$ & -0.137 \\
\hline $\mathrm{S}_{23}$ & 0.056 & $\mathrm{H}_{23}$ & 0.398 & $\mathrm{~S}_{23}$ & -0.018 & $\mathrm{H}_{23}$ & -0.590 \\
\hline $\mathrm{N}_{24}$ & -0.679 & $\mathrm{C}_{22}$ & 0.390 & $\mathrm{~N}_{24}$ & -0.854 & $\mathrm{C}_{22}$ & 0.270 \\
\hline $\mathrm{H}_{25}$ & 0.325 & $\mathrm{C}_{21}$ & -0.298 & $\mathrm{H}_{25}$ & 0.365 & $\mathrm{C}_{21}$ & 0.335 \\
\hline \multirow[t]{7}{*}{$\mathrm{H}_{26}$} & 0.336 & $\mathrm{C}_{20}$ & 0.577 & $\mathrm{H}_{26}$ & 0.372 & $\mathrm{C}_{20}$ & 0.578 \\
\hline & & $\mathrm{O}_{32}$ & -0.396 & & & $\mathrm{O}_{32}$ & -0.577 \\
\hline & & $\mathrm{H}_{24}$ & 0.204 & & & $\mathrm{H}_{24}$ & 0.249 \\
\hline & & $\mathrm{H}_{29}$ & 0.217 & & & $\mathrm{H}_{29}$ & 0.216 \\
\hline & & $\mathrm{H}_{31}$ & 0.2220 & & & $\mathrm{H}_{31}$ & 0.224 \\
\hline & & $\mathrm{C}_{28}$ & 0.610 & & & $\mathrm{C}_{28}$ & -0.596 \\
\hline & & $\mathrm{C}_{22}$ & 0.390 & & & $\mathrm{C}_{22}$ & 0.772 \\
\hline
\end{tabular}

\section{Mullikan and NBO Atomic Charges}

Mullikan and NBO of compounds CQT (3) and CQP (4) were calculated utilized the DFT/B3LYP/6-311G(d) basis set as displayed in Table 5. For the compound the Mullikan charges of CQT(3) showed that $\mathrm{N}_{16}(-0.830)$ and $\mathrm{N}_{24}(-0.679)$ of more negative charges, while the $\mathrm{C}_{9}(0.342)$, also the NBO charges of the same compound seemed at $\mathrm{N}_{16}(-0.566), \mathrm{N}_{24}(-0.854)$ and $\mathrm{C}_{9}(0.148)$ which indicated that the $\mathrm{N}_{16}$ and $\mathrm{N}_{24}$ are the most electrophilic centers of the thiosemicarbazide attached to chloroquinoline moiety and the attached Carbon $\mathrm{C}_{9}$ of chloroquinone which act as nucleophilic susceptibility center which make stability of quinolone ring as displayed in Table 3. Furthermore, the Mullikan and NBO charges of CQP (4) compound showed the most electrophilic negative center of moiety of tetrahydro-6methyl-2-thioxopyrimidin-4(1H)-one moiety which attached to quinolone ring which showed the $\mathrm{N}_{17}(-0.465)$,
$\mathrm{N}_{25}(-0.497), \mathrm{S}_{26}(-0.018)$ and $\mathrm{N}_{27}(-0.681)$ for Mullikan while $\mathrm{N}_{17}(-0.457), \mathrm{N}_{25}(-0.457), \mathrm{S}_{26}(0.212)$ and $\mathrm{N}_{27}(-0.137)$ which meant that the stability of thiopyrimidine moiety with quinolone ring (Ibrahim and Mahmoud, 2009).

\section{Frontier Molecular Orbitals (FMO) and Molecular Electrostatic Potential Maps (ESP)}

The electrical and optical properties can be inferred through chemical reactions and ultraviolet spectra, but FMO is an amazing guideline method for identifying these properties. Time-Dependent Functional Density Theory (TD-DFT) is used for studying FMO principles (Griffith and Orgel, 1957) The highest occupied HOMO molecular orbital acts as an electron donor and the LUMO lowest unoccupied molecular orbital acts as an electron acceptor مرجع. The energy difference between HOMOs and LUMOs related to the biological activity of the molecule (Dennington et al., 2009). Additionally, it 
helps in describing the molecule reactivity and kinetic stability. The high kinetic stability is due to the large energy gap between HOMO-LUMO (Gaussian09, 2009). Figure 6 illustrates the distributions and energy levels of the HOMO, LUMO and orbitals computed at the B3LYP/6-311G (d) level for CQT and CQP. The positive and negative phases were symbolized in red and green colors, respectively. As shown in Fig. 4, the HOMO of compound CQT was localized in the fused of quinolone ring and its LUMO was localized in the Natom of quinolone moiety, the value of the energy gap between the HOMO -LUMO is $(1.49 \mathrm{eV})$. Furthermore, the HOMO and LUMO of CQP was localized on Sulphur atom of tetrahydro-6-methyl-2-thioxopyrimidin$4(1 H)$-one ring with difference in band energy $(3.77 \mathrm{eV})$. This energy gap between HOMO-LUMO indicates the high excitation energies for a lot of excited states and reactivity of these compounds. Moreover, molecular reactivity and biological recognition interactions can be studied by the molecular Electrostatic Potential (ESP) that the nuclei and electrons of a molecule create in the surrounding space (Fukui, 1982). So, the 3-(7chloroquinolin-4-ylamino)-tetrahydro-6-methyl-2-

thioxopyrimidin-4(1H)-one (4) (CQP) designate a certain point then gather this to remaining surface, indicates that there is a uniform distribution of surface contour to fused quinolone, whereas the methyl-2thioxopyrimidin-4(1H)-one moiety contains the $\mathrm{C}=\mathrm{O}$ and $\mathrm{C}=\mathrm{S}$ which acts as electrophilic centers which induced more protonation and gave more reactivity in the biological interaction and in the binding sites of proteins in molecular docking (Schlegel, 1982) as demonstrated in Fig. 4.

\section{Biological Investigation}

\section{Antimalarial Activity}

The investigated compounds CQT (3) and CQP (4) were previously treated against action of $P$. falciparum as displayed in Table 4. The CQT and CQP showed higher antimalarial activity in yield for CQT with $(80 \%$ yield, $\left.\mathrm{IC}_{50}=25.37 \mu \mathrm{g} / \mathrm{mL}\right)$ and CQP $\left(87 \%\right.$ yield, $\mathrm{IC}_{50}=$ $11.92 \mu \mathrm{g} / \mathrm{mL}$ ) as shown in Table 4 and Fig. 5. The preliminary SAR study of CQP has focused on the influence of occurrence methyl-2-thioxopyrimidin$4(1 H)$-one moiety attached to chloroquinoline and make more polarizable of electrons and enhancing their antimalarial activities, while the CQT with low activity due to presence of $\mathrm{NH}_{2}$ attached of $\mathrm{C}=\mathrm{S}$ of thiosemicarbazide and more electrons center which increase the activity (Bawa et al., 2010).

Table 4: The invirto- antimalrial activity of CQT and CQP against plasmodium falciparum

\begin{tabular}{lcr}
\hline Compound & Yield (\%) & IC $_{50}$ \\
\hline CQT & 80 & 25.37 \\
CQP & 87 & 11.92 \\
CQ & 100 & 0.18 \\
\hline
\end{tabular}

CQ: Chlroquinone drug
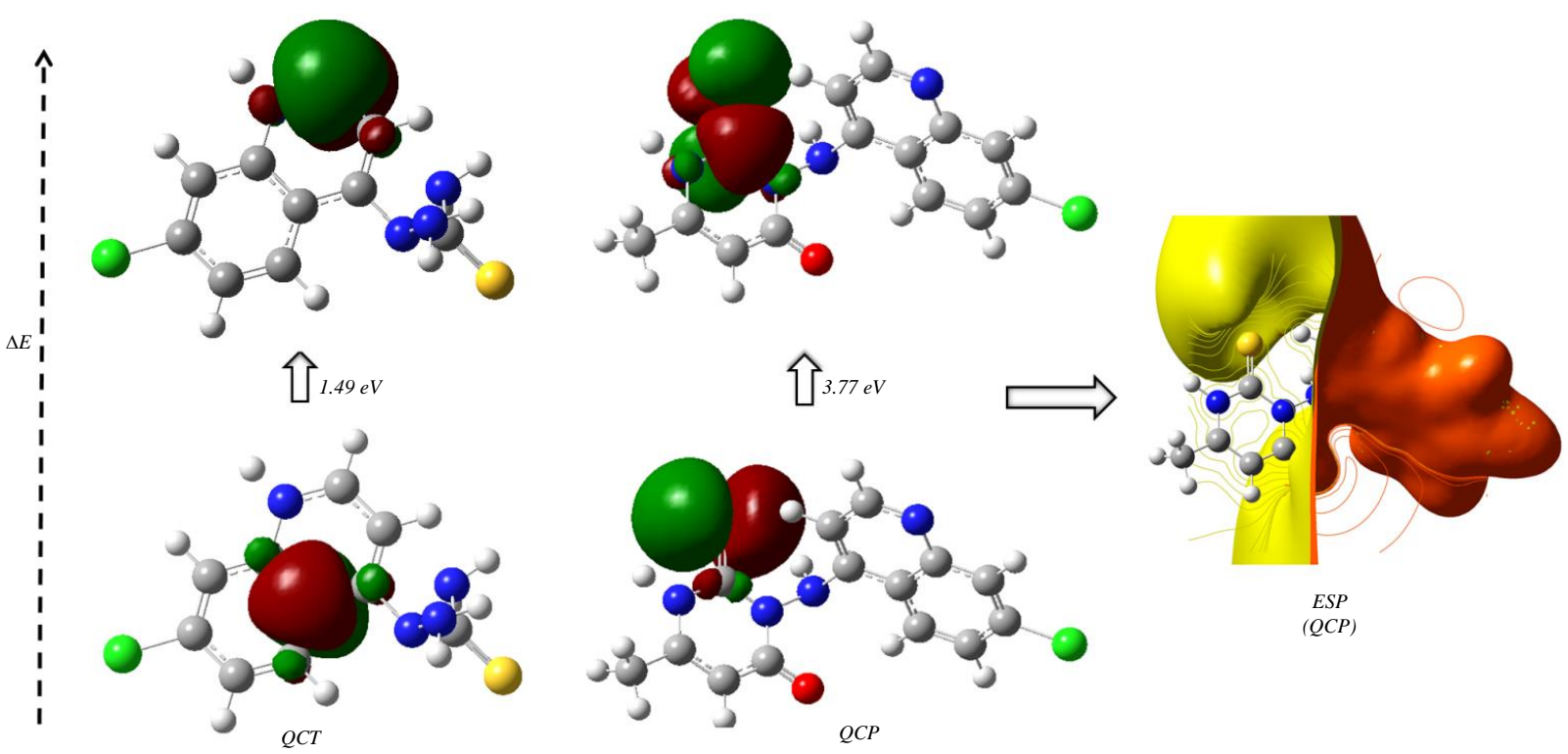

Fig. 4: FMO and ESP of CQT and CQP at (TD-DFT) B3LYP/ 6-311G (d) basis sets 


\section{Molecular Docking Studies}

A molecular modeling study was carried out to explain the cytotoxic activity profile demonstrated by the synthesized compounds. A conformational search using an implicit solvent model was accomplished for the prepared compounds; this was monitored by the refinement of the geometry of local minima through a Quantum-Mechanical (QM) (Morris et al., 2009). Consequently, adaptable docking of the compounds was cultivated in the crystal structure of the (PDBID: 6LU7 Version 2, $2.16 \AA$ resolution, CQP (4) was stimulated with functions as a homo dimer (Jin et al., 2020) and leads that target main protease (Mpro) of SARS-CoV-2: Mpro is a key enzyme of coronaviruses and has a pivotal role in mediating viral replication and transcription, making it an attractive drug target for SARS-CoV-25,6 (Jo et al., 2020). The docking simulation of CQT and CQP with (PDBID: 6lu7) as shown in Table 5 and Fig. 6 to evaluate the binding interaction energy and the distance between protein's and it was shown that the (PDBID: 6lu7) attached to CQP with $(\Delta E=-14.4383 \mathrm{Kcal} / \mathrm{mol}, 2.337 \AA)$ with Hydrogen bonding with amino acid Pro132 of $\mathrm{NH}$ group rather than the CQT attched with binding energy $(\Delta E=-12.2755 \mathrm{Kcal} / \mathrm{mol}, 3.576 \AA)$ and attched to chloroquinoline with Phe294. We identified a mechanismbased inhibitor (N3) through docking stimulation and determined that the crystal structure of Mpro of SARSCoV-2 in complex with CQT and CQP. Through a combination of structure-based virtual and highthroughput screening, Furthermore, crystal structure of a protein of unknown function TA1206 from thermoplasma acidophilum (PDBID: 1qw2O) (Pathare et al., 2017) which contain of single unique chain 1qw2(A) (102 residues long) (Pathare et al., 2017). The compounds CQT and CQP were docked with (PDBID: 1qw2) with binding interaction energy $(\Delta E=-6.66,-13.61 \mathrm{kcal} / \mathrm{mol})$; respectively and with short bond distance og CQP with $2.51 \AA$ with NH between chloroquinone and tetrahydro-6methyl-2-thioxopyrimidin-4(1H)-one ring which confirmed this H-proton the most active in mobilization of electrons and increase the biological evaluation of Compound $\mathrm{CQP}$ rather than CQT which its docking ability with (PDBID: 1qw2) with $\mathrm{NH}_{2}$ groups and bond distance 3.21 $\mathrm{A}$ as shown in Table 5 and Fig. 6 .

Table 5: Docking of CQT(3) and CQP(4) with (PDB ID: 6lu7) and (PDBID:1q2w):

\begin{tabular}{llll}
\hline Compound & Energy affinity $(\mathrm{kcal} / \mathrm{mol})$ & Distance $(\AA)$ & Amino acids \\
\hline PDBID: 6lu7 & & & \\
CQT & -12.2755 & 3.576 & Gln110,Ph294,Asn 151, Ser158, Asp153 and Asn 133 \\
CQP & -14.4383 & 2.337 & Pro132, Thr196, Asp153, Asn 133, Glu240 and Thr 198 \\
PDBID: 1q2w(Chain A) & & & \\
CQT & -6.6601 & 3.21 & Pro108, His 246, GlnA107 \\
CQP & -13.6197 & 2.51 & Pro A108, GluA240,HisA246 and Leu202 \\
\hline
\end{tabular}

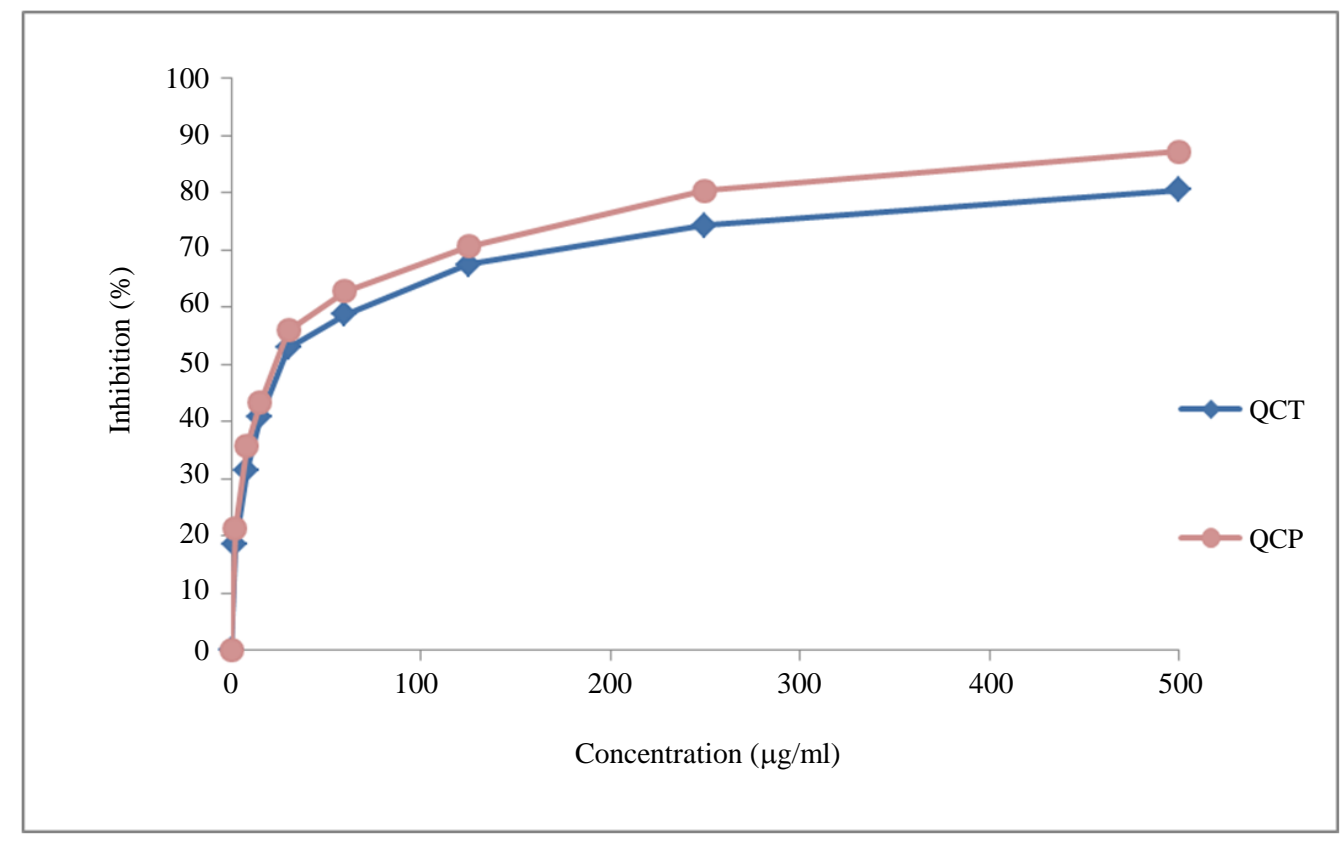

Fig. 5: The antimalarial concentration of CQT (3) and CQP (4) 

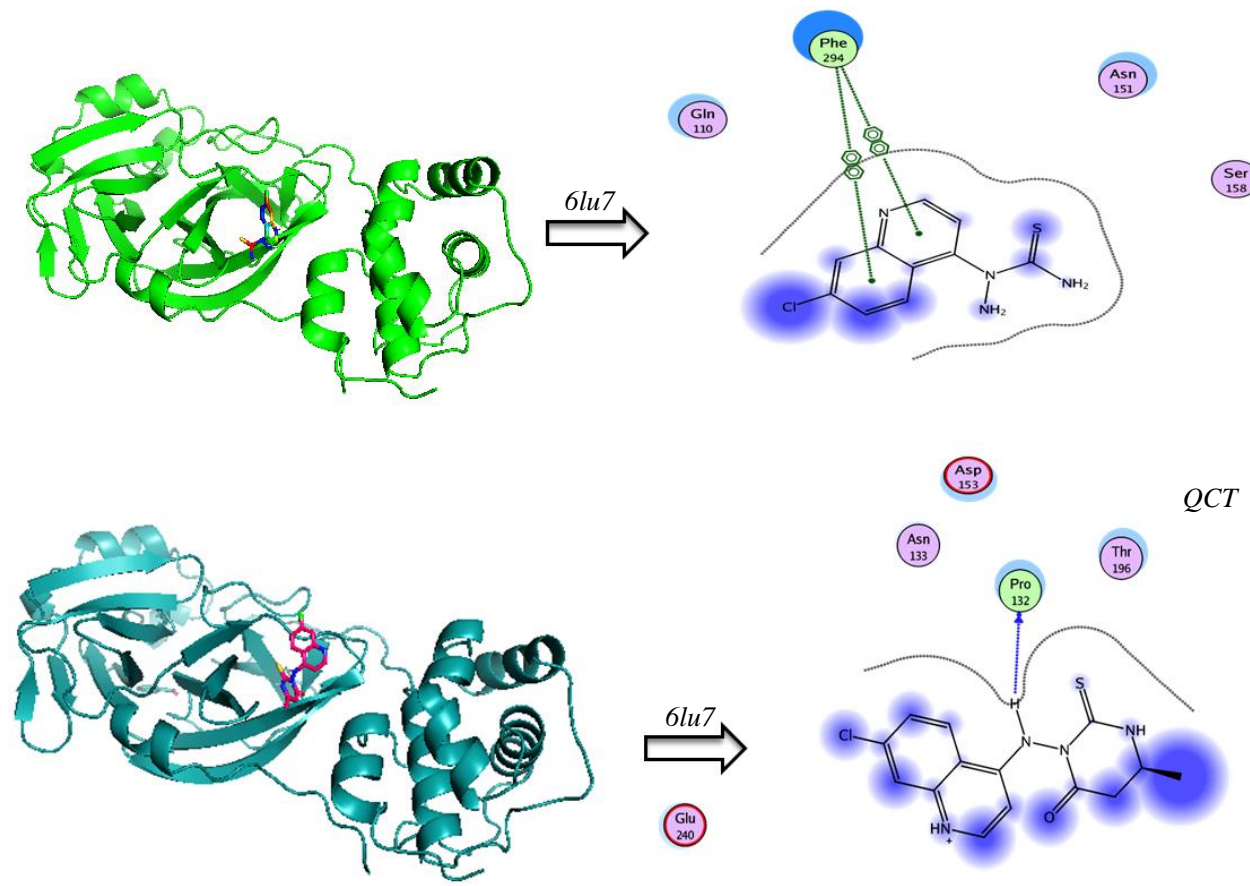

$Q C P$

Fig. 6: Binding docking modes of CQT, CQP with (a) PDBID: 6lu7 and (b) PDBID:1qw2; respectively

\section{Experimental}

\section{General Procedure}

Melting points were measured with a Gallen Kamp melting point apparatus. Silica-gel-coated aluminum plates used to test the purity of the compounds. Infrared spectra $\left(\lambda-\mathrm{cm}^{-1}\right)$ were recorded on Bruker Vector (Germany) and on Mattson FT-IR 1000 (Cairo University, Egypt), using $\mathrm{KBr}$ disks. ${ }^{1} \mathrm{H}$ NMR spectra were recorded on Gemini $300 \mathrm{MHz},{ }^{13} \mathrm{CNMR}$ spectrometer, in DMSO- $\mathrm{d}_{6}$ using dimethyl sulfoxide as a solvent and tetramethylsilane (TMS) as an internal standard (Chemical shift in $\delta$, ppm); ${ }^{13} \mathrm{C}$ NMR spectra were recorded on Gemini $50 \mathrm{MHz}$ NMR spectrometer. Mass spectra were measured on GCQ Finnigan MAT and Elemental analyses were performed at the micro analytical Center, Cairo University, Giza, Egypt. Biological activity was determined in a laboratory by the Regional Center for Mycology and Biotechnology (RCMB), Al-Azhar University, Cairo, Egypt. All the chemicals were purchased from Sigma-Aldrich.

Synthesis of 2-(7-Chloroquinolin-4yl)Thiosemicarbazide (3)

An ethanolic solution of 4,7-dichloroquinoline (1) (0.196 g, $0.01 \mathrm{~mol})$ with thiosemicarbazide (2) (0.096,
0.01 mole) were stirred in ultrasonic apertures at room temperature for $30 \mathrm{~min}$, then monitoring TLC to know the reaction was finished then add $\mathrm{CH}_{3} \mathrm{Cl}$ and washed with solution $\mathrm{NaOH}$ solution to afford the organic layer and aqueous layer. Then the crude poured on ice bath to afford 2-(7-chloroquinolin-4-yl)thiosemicarbazide (3): yellow soild which was washed with ether and dried. Yield: $80 \%$; $\mathrm{mp} 278-280^{\circ} \mathrm{C}, \mathrm{Rf}=0.38(1: 2 \mathrm{EtOAc}-$ petroleum ether); IR $(\mathrm{KBr}) \mathrm{cm}^{-1}: 3390-3266\left(\mathrm{NH}_{2}\right)$, $3150(\mathrm{NH}), 1615(\mathrm{C}=\mathrm{N}), 1585\left(\mathrm{NH}_{\text {bend }}\right), 1235(\mathrm{C}-\mathrm{N})$, $1212(\mathrm{C}=\mathrm{S}), 758(\mathrm{C}-\mathrm{Cl}) ;{ }^{1} \mathrm{H}$ NMR(300 MHz, DMSO$\left.\mathrm{d}_{6}\right) \delta 8.89(\mathrm{~d}, J=5.48 \mathrm{~Hz}, 1 \mathrm{H}, \mathrm{N}=\mathrm{CH}$, quinoline $)$, $8.57(\mathrm{~d}, J=8.48 \mathrm{~Hz}, 1 \mathrm{H}, \mathrm{Ar}-\mathrm{H}), 8.54(\mathrm{~s}, 1 \mathrm{H}, \mathrm{Ar}-\mathrm{H})$, 8.26(d, $J=8.43 \mathrm{~Hz}, 1 \mathrm{H}, \mathrm{Ar}-\mathrm{H}), 8.20$ (s, 2H, NH2), 8.10 (s, 1H, ArC-NH), 7.93(d, $J=5.42 \mathrm{~Hz}, 1 \mathrm{H}, \mathrm{Ar}-\mathrm{H}), 7.86$ (s, 1H, NH); 13C NMR(50 MHz, DMSO-d 6 ) 181.22, 158.22, 155.72, 149.02, 148.14, 138.50, 128.42, 127.65, 120.03, 18.34. Mass spectra $\left(\mathrm{M}^{ \pm}\right) \mathrm{m} / \mathrm{z}=252$. Anal. Calcd for $\mathrm{C}_{10} \mathrm{H}_{9} \mathrm{ClN}_{4} \mathrm{~S}$ : C,47.53; H, 3.59; N, 22.17. Found: C, 47.33; H, 3.52; N, 22.09\%.

Reactivity of 2-(7-Chloroquinolin-4yl)Thiosemicarbazide (3) with Ethyl Acetoacetate

Ultrasonic reaction of compound (3) $(0.25 \mathrm{~g}, 0.01$ mol) with ethylacetoacetate $(2 \mathrm{~mL}, 0.01 \mathrm{~mol})$ in water bath for $40 \mathrm{~min}$ at $90^{\circ} \mathrm{C}$, the solid product was formed 
and filtered to afford the corresponding product (4) and crystallized with EtOH 3-(7-chloroquinolin-4-ylamino)tetrahydro-6-methyl-2-thioxopyrimidin-4(1H)-one (4): Brown crystals which was washed with ether and dried. Yield: $87 \%$; $\mathrm{mp} 250-252^{\circ} \mathrm{C} \mathrm{Rf}=0.48(1: 2 \mathrm{EtOAc}-$ petroleum ether); IR (KBr) $\mathrm{cm}^{-1}: 3110(\mathrm{NH}), 1674(\mathrm{C}=$ $\mathrm{O}), 1618(\mathrm{C}=\mathrm{N}), 1587$ (NH bend), $1237(\mathrm{C}-\mathrm{N}), 1211(\mathrm{C}$ $=\mathrm{S}), 760(\mathrm{C}-\mathrm{Cl}) ; 1 \mathrm{H} \mathrm{NMR}\left(300 \mathrm{MHz}, \mathrm{DMSO}-\mathrm{d}_{6}\right): \delta 8.20$ (d, $J=5.38 \mathrm{~Hz},{ }^{1} \mathrm{HN}=\mathrm{CH}$ quinoline) $8.10\left(\mathrm{~s},{ }^{1} \mathrm{H}, \mathrm{NH}\right)$; 7.85 (d, J = 8.32 Hz, ${ }^{1} \mathrm{H}$, Ar- H), 7.82 (s, ${ }^{1} \mathrm{H}$, Ar- H), 7.18 $(\mathrm{d}, J=5.38 \mathrm{~Hz}, 1 \mathrm{H}, \mathrm{Ar}-\mathrm{H}), 7.15\left(\mathrm{~d}, J=5.38 \mathrm{~Hz},{ }^{1} \mathrm{H}, \mathrm{Ar}-\right.$ $\mathrm{H}), 2.50\left(\mathrm{~s}, 2 \mathrm{H}, \mathrm{CH}_{2}\right), 2.17\left(\mathrm{~s}, 3 \mathrm{H}, \mathrm{CH}_{3}\right) ;{ }^{13} \mathrm{C}$ NMR $(50$ $\mathrm{MHz}$, DMSO-d 6 ) $\delta 181.36,166.23,156.96,151.13$, $149.15,141.30,138.57,122.23,119.52,118.17,115.31$, 113.80, 91.21, 48.79. Mass spectra $\left(\mathrm{M}^{ \pm}\right) \mathrm{m} / \mathrm{z}=318$. Anal. Calcd.for $\mathrm{C}_{14} \mathrm{H}_{11} \mathrm{~N}_{4} \mathrm{ClOS}: \mathrm{C}, 52.75 ; \mathrm{H}, 3.48 ; \mathrm{N}$, 17.58. Found: C, 52.91; H, 3.32; N,17.71\%.

\section{Determination of Antimalarial Activity}

Cultures of $P$. falciparum were measured according to a protocol from (Moloney et al., 1990; Trager and Williams, 1992). The percentage of Inhibition was studied the $50 \%$ inhibitory concentrations $\left(\mathrm{IC}_{50}\right)$ was measured and the data got from the inhibitordependent concentration growth curves were registered into plots with nonlinear retreating analysis from $\mathrm{y}$-axis (inhibition \%) to $\mathrm{x}$-axis (inhibitor concentration) (Singh et al., 1997) $P$. falciparum isolate NF54 was maintained in continuous culture with gentamicin $(40 \mu \mathrm{g} / \mathrm{mL})$ in Petri dishes $(5 \mathrm{~cm}$ in diameter) with a gaseous phase of $90 \% \mathrm{~N}_{2}, 5 \% \mathrm{O}_{2}$ and $5 \% \mathrm{CO}_{2}$, according to a protocol from (Moloney et al., 1990; Trager and Williams, 1992) P. falciparum parasites were cultured in human erythrocytes (blood group $\mathrm{A}+$ at $10 \%(\mathrm{v} / \mathrm{v})$ hematocrit) in RPMI 1640 medium (Sigma) supplemented with $25 \mathrm{mM}$ HEPES, 20 $\mathrm{mM}$ sodium bicarbonate and $10 \%$ heat-inactivated human A+ plasma. The culture was routinely monitored through Geimsa staining of the thin blood smears. The parasitemia of the infected erythrocytes was determined in Giemsa-stained smears by light microscopy. Parasitemias and morphological changes detected in the cultures were scored visually with a 100-fold oilimmersion objective, counting at least 1000 erythrocytes to determine the percentage of the infected erythrocytes (Kaiser et al., 2003). Antimalarial activity assay: The experiments were performed in 96-well culture plates (Nunc); compounds were tested at two-fold dilutions in a dose-titration range of 500 to $2 \mu \mathrm{M}$. One hundred microliters of infected human red blood cell suspension ( $1 \%$ parasitemia, $4 \%$ hematocrit), with more than $90 \%$ of ring forms, were added to each well containing $100 \mathrm{~mL}$ of extracts pre-diluted in RPMI-1640. Test plates were incubated for $48 \mathrm{~h}$. Parasite multiplication was determined microscopically after Giemsa staining and expressed as a percentage of the controls without test compounds. A drug-free control (methanol/water $50: 50 \% \mathrm{v}, \mathrm{v})$ was used in all experiments and CQ (0.01 $\mu \mathrm{M})$ was used as the standard reference drug. Parasitemia and stage distribution were estimated as triplicates daily from Giemsa-stained smears by counting 1000 erythrocytes (Noedl et al., 2002).

\section{Computational Procedures}

Calculations of DFT with a hybrid functional B3LYP (Becke's three-parameter hybrid functional using the BLYP correlation functional) with the 6-311G(d) basis set and Hartree-Fock calculations with the 6-311G(d) basis set using the Berny method (Jamróz, 2013), were performed with the Gaussian 09 W program (Ditchfield, 1972). No symmetry constraints were applied during the geometry optimization. The harmonic vibrational frequencies were calculated at the same level of theory for the optimized structures to prove the optimized structures as true minimums and confirm that no imaginary frequency occurs. The wideranging assignments of the vibrational modes were accomplished on the basis of the Potential Energy Distribution (PED), calculated using Vibrational Energy Distribution Analysis (VEDA) program (Foresman and Frish, 1996).

\section{Molecular Docking}

The molecular model of innovative sulfonamide derivatives was fabricated using standard bond lengths and angles, with the AutoDock Vina and detected by Discovery Studio Client (version 4.2). Following geometry optimization, a systematic conformational examine was supported out to an RMS gradient of 0.01 $\AA$, with energy minimization of the resultant conformations employing the Confirmation Examination module implemented in Auto Dock Vina. The experimental Structure of Mprofrom SARS-CoV-2 and discovery of its inhibitors (PDBID: 6lu7) (Jo et al., 2020) and Crystal structure of a protein of unknown function ta1206 from thermoplasma acidophilum (PDBID: 1qw2) (Pathare et al., 2017). Missing hydrogens were added to the enzyme and partial charges were considered. After removing the co-crystallized inhibitor, validation monitored by docking of the compounds were carried out by AutoDock Vina and viewed by Discovery Studio Client (version 4.2) (Morris et al., 2009). The goal protein was kept inflexible, while the ligands were disappeared permitted to determine the conformational space exclusive the enzyme cavity; Twenty dispersed docking simulations were run via default parameters and the confirmations were designated constructed on the arrangement of total statistics, $E$ conformation and appropriate with the relevant amino acids in the binding pocket. 


\section{Conclusion}

In this study, the synthesis of some novel chloroquinoline derivative using green methodology, the synthesized compounds were exhibited high antimalarial activities. The SAR relationship related to the most active compound was CQP (4) with $\mathrm{IC}_{50}=11.92 \mu \mathrm{g} / \mathrm{ml}$ and due to cyclized tetrahydro-6-methyl-2-thioxopyrimidin-4(1H)one ring attached to chlorine ring. The optimized molecular structure of compounds CQT and CQP utilizing of DFT/B3LYP/6-311G(d) and HF/6-311G(d) basis's set, approves their stability and the geometric parameters suggestions between the calculated and the experimental data values indicate that B3LYP basis set is better than the $\mathrm{HF}$ method in approximating bond lengths and in evaluating energy, Mullikan and NBO charges. Furthermore, the CQT and CQP were docked against (PDB ID: 6lu7) and (PDBID: 1q2w) and showed the NHhydrogen: $2.337 \AA$ of CQP with (Mpro) of SARS-CoV-2. So for further biological investigation we will test these compounds and other quinolone derivatives in treatment of SARS-Covid-19.

\section{Acknowledgment}

The authors thanks Departement of chemistry Faculty of Women of Arts, Science and Education, Ain Shams University and Departments of Green Chemistry, National Research Center for scientific contribution

\section{Author's Contributions}

Asmaa Aboelnaga: Preparation of organic compounds and main corresponding author.

Asmaa M. Fahim: Make theoretical studies and elucidation the main idea of manuscript.

Taghreed H. EL-Sayed: Synthesis the organic compounds and revise the manuscript.

\section{Ethics}

This article is original and contains unpublished material. The corresponding author confirms that all of the other authors have read and approved the manuscript and no ethical issues involved.

\section{References}

Aboelnaga, A., \& EL-Sayed, T. H. (2018). Click synthesis of new 7-chloroquinoline derivatives by using ultrasound irradiation and evaluation of their biological activity. Green Chemistry Letters and Reviews, 11(3), 254-263.

Adem, S., Eyupoglu, V., Sarfraz, I., Rasul, A., \& Ali, M. (2020). Identification of potent COVID-19 main protease (Mpro) inhibitors from natural polyphenols: An in silico strategy unveils a hope against CORONA.
Akl, E. M., Dacrory, S., Abdel-Aziz, M. S., Kamel, S., \& Fahim, A. M. (2020). Preparation and characterization of novel antibacterial blended films based on modified carboxymethyl cellulose/phenolic compounds. Polymer Bulletin, 1-25.

Arif, R., Rana, M., Yasmeen, S., Khan, M. S., Abid, M., \& Khan, M. S. (2020). Facile synthesis of chalcone derivatives as antibacterial agents: Synthesis, DNA binding, molecular docking, DFT and antioxidant studies. Journal of Molecular Structure, 1208, 127905.

Atkins, P., \& De Paula, J. (2011). Physical chemistry for the life sciences. Oxford University Press, USA.

Bawa, S., Kumar, S., Drabu, S., \& Kumar, R. (2010). Structural modifications of quinoline-based antimalarial agents: Recent developments. Journal of Pharmacy and Bioallied Sciences, 2(2), 64.

Bzówka, M., Mitusinska, K., Raczynska, A., Samol, A., Tuszynski, J. A., \& Góra, A. (2020a). Molecular Dynamics Simulations Indicate the COVID-19 Mpro Is Not a Viable Target for Small-Molecule Inhibitors Design. bioRxiv.

Bzówka, M., Mitusińska, K., Raczyńska, A., Samol, A., Tuszyński, J. A., \& Góra, A. (2020b). Structural and Evolutionary Analysis Indicate That the SARSCoV-2 Mpro Is a Challenging Target for SmallMolecule Inhibitor Design. International journal of molecular sciences, 21(9), 3099.

Chen, Y. W., Yiu, C. P. B., \& Wong, K. Y. (2020). Prediction of the SARS-CoV-2 (2019-nCoV) 3Clike protease (3CL pro) structure: virtual screening reveals velpatasvir, ledipasvir and other drug repurposing candidates. F1000Research, 9.

Dacrory, S., \& Fahim, A. M. (2020). Synthesis, antiproliferative activity, computational studies of tetrazole cellulose utilizing different homogenous catalyst. Carbohydrate Polymers, 229, 115537.

Dennington, R., Keith, T., \& Millam, J. G. (2009). version 5; Semichem Inc. Shawnee Mission, KS.

Ditchfield, R. (1972). Molecular orbital theory of magnetic shielding and magnetic susceptibility. The Journal of Chemical Physics, 56(11), 5688-5691.

dos Santos Chagas, C., Fonseca, F. L., \& Bagatin, I. A. (2019). Quinoline-derivative coordination compounds as potential applications to antibacterial and antineoplasic drugs. Materials Science and Engineering: C, 98, 1043-1052.

Fahim, A. M., \& Shalaby, M. A. (2019). Synthesis, biological evaluation, molecular docking and DFT calculations of novel benzenesulfonamide derivatives. Journal of Molecular Structure, 1176, 408-421.

Fahim, A. M., Wasiniak, B., \& Łukaszewicz, J. P. (2020). Molecularly Imprinted Polymer and Computational Study of (E)-4-(2-cyano-3(dimethylamino) acryloyl) benzoic Acid from Poly (ethylene terephthalate) Plastic Waste. Current Analytical Chemistry, 16(2), 119-137. 
Fahim, A., \& Ismael, E. (2019). Synthesis, Antimicrobial Activity and Quantum Calculations of Novel Sulphonamide Derivatives. Egyptian Journal of Chemistry, 62(8), 1427-1440.

Farag, A. M., \& Fahim, A. M. (2019). Synthesis, biological evaluation and DFT calculation of novel pyrazole and pyrimidine derivatives. Journal of Molecular Structure, 1179, 304-314.

Fehr, A. R., \& Perlman, S. (2015). Coronaviruses: an overview of their replication and pathogenesis. In Coronaviruses (pp. 1-23). Humana Press, New York.

Foresman, J., \& Frish, E. (1996). Exploring chemistry. Gaussian Inc., Pittsburg, USA.

Fukui, K. (1982). Role of frontier orbitals in chemical reactions. science, 218(4574), 747-754.

Gaussian09, R. A. (2009). 1, mj frisch, gw trucks, hb schlegel, ge scuseria, ma robb, jr cheeseman, g. Scalmani, v. Barone, b. Mennucci, ga petersson et al., gaussian. Inc., Wallingford CT, 121, 150-166.

Gimeno, A., Ojeda-Montes, M. J., Tomás-Hernández, S., Cereto-Massagué, A., Beltrán-Debón, R., Mulero, M., ... \& Garcia-Vallvé, S. (2019). The light and dark sides of virtual screening

Griffith, J. S., \& Orgel, L. E. (1957). Ligand-field theory. Quarterly Reviews, Chemical Society, 11(4), 381-393.

Grimstein, M., Yang, Y., Zhang, X., Grillo, J., Huang, S. M., Zineh, I., \& Wang, Y. (2019). Physiologically based pharmacokinetic modeling in regulatory science: an update from the US Food and Drug Administration's Office of Clinical Pharmacology. Journal of pharmaceutical sciences, 108(1), 21-25.

Guo, Y. R., Cao, Q. D., Hong, Z. S., Tan, Y. Y., Chen, S. D., Jin, H. J., ... \& Yan, Y. (2020). The origin, transmission and clinical therapies on coronavirus disease 2019 (COVID-19) outbreak-an update on the status. Military Medical Research, 7(1), 1-10.

Hagar, M., Chaieb, K., Parveen, S., Ahmed, H. A., \& Alnoman, R. B. (2020). N-alkyl 2-pyridone versus O-alkyl 2-pyridol: Ultrasonic synthesis, DFT, docking studies and their antimicrobial evaluation. Journal of Molecular Structure, 1199, 126926.

Hema, R., Parthasarathi, V., Ravikumar, K., Nallu, M., \& Sarkunam, K. (2015). Acta Crystallographica Section E Structure Reports Online ISSN 1600-5368.

Hosseini, F. S., \& Amanlou, M. (2020). Simeprevir, potential candidate to repurpose for coronavirus infection: virtual screening and molecular docking study.

Ibrahim, M., \& Mahmoud, A. A. (2009). Computational notes on the reactivity of some functional groups. Journal of Computational and Theoretical Nanoscience, 6(7), 1523-1526.

Jamróz, M. H. (2013). Vibrational energy distribution analysis (VEDA): scopes and limitations. Spectrochimica Acta Part A: Molecular and Biomolecular Spectroscopy, 114, 220-230.
Jin, Z., Du, X., Xu, Y., Deng, Y., Liu, M., Zhao, Y., ... \& Duan, Y. (2020). Structure of Mpro from COVID-19 virus and discovery of its inhibitors. bioRxiv. Preprint.

Jo, S., Kim, S., Shin, D. H., \& Kim, M. S. (2020). Inhibition of SARS-CoV 3CL protease by flavonoids. Journal of enzyme inhibition and medicinal chemistry, 35(1), 145-151.

Kaiser, A., Gottwald, A., Maier, W., \& Seitz, H. M. (2003). Targeting enzymes involved in spermidine metabolism of parasitic protozoa-a possible new strategy for anti-parasitic treatment. Parasitology Research, 91(6), 508-516.

Khaerunnisa, S., Kurniawan, H., Awaluddin, R., Suhartati, S., \& Soetjipto, S. (2020). Potential inhibitor of COVID-19 main protease (Mpro) from several medicinal plant compounds by molecular docking study. Prepr. doi10, 20944, 1-14.

Kong, R., Yang, G., Xue, R., Liu, M., Wang, F., Hu, J., ... \& Chang, S. (2020). COVID-19 Docking Server: An interactive server for docking small molecules, peptides and antibodies against potential targets of COVID-19. arXiv preprint arXiv:2003.00163.

Kwofie, S. K., Broni, E., Dankwa, B., Enninful, K. S., Teye, J., Davidson, C. R., ... \& Miller, W. A. (2020). Review of Atypical Organometallic Compounds as Antimalarial Drugs. Journal of Chemistry, 2020.

Liu, J., Cao, R., Xu, M., Wang, X., Zhang, H., Hu, H., ... \& Wang, M. (2020). Hydroxychloroquine, a less toxic derivative of chloroquine, is effective in inhibiting SARS-CoV-2 infection in vitro. Cell discovery, 6(1), 1-4.

Liu, X., \& Wang, X. J. (2020). Potential inhibitors against 2019-nCoV coronavirus M protease from clinically approved medicines. Journal of Genetics and Genomics, 47(2), 119.

Ludwig, S., \& Zarbock, A. (2020). Coronaviruses and SARS-CoV-2: A Brief Overview. Anesthesia and Analgesia.

Mirza, M. U., \& Froeyen, M. (2020). Structural elucidation of SARS-CoV-2 vital proteins: Computational methods reveal potential drug candidates against main protease, Nsp12 polymerase and Nsp13 helicase. Journal of Pharmaceutical Analysis.

Moloney, M. B., Pawluk, A. R., \& Ackland, N. R. (1990). Plasmodium falciparum growth in deep culture. Transactions of the Royal Society of Tropical Medicine and Hygiene, 84(4), 516-518.

Morris, G. M., Huey, R., Lindstrom, W., Sanner, M. F., Belew, R. K., Goodsell, D. S., \& Olson, A. J. (2009). AutoDock4 and AutoDockTools4: Automated docking with selective receptor flexibility. Journal of computational chemistry, 30(16), 2785-2791.

Noedl, H., Wernsdorfer, W. H., Miller, R. S., \& Wongsrichanalai, C. (2002). Histidine-rich protein II: a novel approach to malaria drug sensitivity testing. Antimicrobial agents and chemotherapy, 46(6), 1658-1664 
Pathare, G. R., Nagy, I., Hubert, A., Thomas, D. R., \& Bracher, A. (2017). Crystal structure of the Thermoplasma acidophilum protein Ta1207. Acta Crystallographica Section F: Structural Biology Communications, 73(6), 328-335.

Rodriguez-Morales, A. J., Bonilla-Aldana, D. K., Balbin-Ramon, G. J., Rabaan, A. A., Sah, R., PanizMondolfi, A., ... \& Esposito, S. (2020). History is repeating itself: Probable zoonotic spillover as the cause of the 2019 novel Coronavirus Epidemic. Infez Med, 28(1), 3-5.

Schlegel, H. B. (1982). Optimization of equilibrium geometries and transition structures. Journal of Computational Chemistry, 3(2), 214-218.

Singh, S., Puri, S. K., Singh, S. K., Srivastava, R., Gupta, R. C., \& Pandey, V. C. (1997). Characterization of Simian Malarial Parasite (Plasmodium knowlesi)-induced Putrescine Transport in Rhesus Monkey Erythrocytes a novel putrescine conjugate arrests in vitro growth of simian malarial parasite (plasmodium knowlesi) and cures multidrug resistant murine malaria (plasmodium yoelii) infection in vivo. Journal of Biological Chemistry, 272(21), 13506-13511.

Sjödin, H., Wilder-Smith, A., Osman, S., Farooq, Z., \& Rocklöv, J. (2020). Only strict quarantine measures can curb the coronavirus disease (COVID-19) outbreak in Italy, 2020. Eurosurveillance, 25(13), 2000280.

Steardo, L., Steardo Jr, L., Zorec, R., \& Verkhratsky, A. (2020). Neuroinfection may contribute to pathophysiology and clinical manifestations of COVID-19. Acta Physiologica, e13473.

Tang, B., He, F., Liu, D., Fang, M., Wu, Z., \& Xu, D. (2020). AI-aided design of novel targeted covalent inhibitors against SARS-CoV-2. bioRxiv.

Ton, A. T., Gentile, F., Hsing, M., Ban, F., \& Cherkasov, A. (2020). Rapid identification of potential inhibitors of SARS-CoV-2 main protease by deep docking of 1.3 billion compounds. Molecular informatics.

Trager, W., \& Williams, J. (1992). Extracellular (axenic) development in vitro of the erythrocytic cycle of Plasmodium falciparum. Proceedings of the National Academy of Sciences, 89(12), 5351-5355.

Trott, O., \& Olson, A. J. (2010). AutoDock Vina: improving the speed and accuracy of docking with a new scoring function, efficient optimization and multithreading. Journal of computational chemistry, 31(2), 455-461.

Wu, F., Zhao, S., Yu, B., Chen, Y. M., Wang, W., Song, Z. G., ... \& Yuan, M. L. (2020a). A new coronavirus associated with human respiratory disease in China. Nature, 579(7798), 265-269.
Wu, C., Liu, Y., Yang, Y., Zhang, P., Zhong, W., Wang, Y., ... \& Zheng, M. (2020b). Analysis of therapeutic targets for SARS-CoV-2 and discovery of potential drugs by computational methods. Acta Pharmaceutica Sinica B.

Xu, Z., Peng, C., Shi, Y., Zhu, Z., Mu, K., Wang, X., \& Zhu, W. (2020). Nelfinavir was predicted to be a potential inhibitor of 2019-nCov main protease by an integrative approach combining homology modelling, molecular docking and binding free energy calculation. BioRxiv.

Yamamoto, K., Sawanishi, H., \& Miyamoto, K. I. (1998). Structure-activity relationships of alkylxanthine inhibitors of phosphodiesterase IV isoenzyme. Biological and Pharmaceutical Bulletin, 21(4), 356-359.

Yang, P., \& Wang, X. (2020). COVID-19: a new challenge for human beings. Cellular \& molecular immunology, 17(5), 555-557.

Yang, W., \& Parr, R. G. (1985). Hardness, softness and the fukui function in the electronic theory of metals and catalysis. Proceedings of the National Academy of Sciences, 82(20), 6723-6726.

Yao, X., Ye, F., Zhang, M., Cui, C., Huang, B., Niu, P., ... \& Zhan, S. (2020). In vitro antiviral activity and projection of optimized dosing design of hydroxychloroquine for the treatment of severe acute respiratory syndrome coronavirus 2 (SARSCoV-2). Clinical Infectious Diseases.

Yoshino, R., Yasuo, N., \& Sekijima, M. (2020). Identification of key interactions between SARSCoV-2 Main Protease and inhibitor drug candidates.

Zhao, S., Lin, Q., Ran, J., Musa, S. S., Yang, G., Wang, W., ... \& Wang, M. H. (2020). Preliminary estimation of the basic reproduction number of novel coronavirus (2019-nCoV) in China, from 2019 to 2020: A data-driven analysis in the early phase of the outbreak. International journal of infectious diseases, 92, 214-217.

Zhou, P., Yang, X. L., Wang, X. G., Hu, B., Zhang, L., Zhang, W., ... \& Chen, H. D. (2020). A pneumonia outbreak associated with a new coronavirus of probable bat origin. nature, 579(7798), 270-273. 